\title{
Subgap states in superconducting islands
}

\author{
Luka Pavešić, ${ }^{1,2}$ Daniel Bauernfeind, ${ }^{3}$ and Rok Žitko ${ }^{1,2}$ \\ ${ }^{1}$ Jožef Stefan Institute, Jamova 39, SI-1000 Ljubljana, Slovenia \\ ${ }^{2}$ Faculty of Mathematics and Physics, University of Ljubljana, Jadranska 19, SI-1000 Ljubljana, Slovenia \\ ${ }^{3}$ Center for Computational Quantum Physics, Simons Foundation Flatiron Institute, New York, New York 10010, USA
}

(Dated: October 4, 2021)

\begin{abstract}
We study an interacting quantum dot in contact with a superconducting island described by the Richardson model with a Coulomb repulsion term controlling the number of electrons on the island. This Hamiltonian admits a compact matrix-product-operator representation and can be efficiently and accurately solved using the density-matrix renormalization group. We systematically explore the effects of the charging energy $E_{c}$. For $E_{c}$ comparable to the superconducting gap $\Delta$, the subgap states are stabilized by the combination of Kondo exchange coupling and charge redistribution driven by the Coulomb interaction. The subgap states exist for both even and odd superconductor ground-state occupancy, but with very distinctive excitation spectra in each case. The spectral peaks are not symmetric with respect to the chemical potential and may undergo discontinuous changes as a function of gate voltages.
\end{abstract}

The study of long-lived excited states inside the bulk spectral gap of superconductors (subgap states for short), induced by impurities and interfaces, drives the development of technologically important quantum devices. For example, the Yu-Shiba-Rusinov (YSR) states that result from the exchange interaction which binds a Bogoliubov quasiparticle at the magnetic impurity site [1-4] are instrumental in realizing topological superconductivity with Majorana edge modes [5]. The excellent understanding of YSR states rests on a theoretical description based on the Anderson impurity model with a superconducting (SC) bath described by the Bardeen-Cooper-Schrieffer (BCS) mean-field Hamiltonian [6-9], which can be tackled using modern impurity solvers [10-16].

A recent development are devices with the SC material epitaxially evaporated on the nanowire hosting the impurity (quantum dot, QD) [17, 18]. The small SC island in these devices has a considerable charging energy $E_{c}$ and strong even-odd occupancy effects [19-22] that require an appropriate description [23-29]. This is similar to SC metallic grains [30-34] described by the Richardson model, a charge-conserving Hamiltonian with pairing between the time-reversal-invariant pairs of states in the orbital basis, which is the appropriate generalization of the BCS pairing Hamiltonian to a situation with no translation invariance [35]. For weak to moderate pairing and a dense set of levels, the Richardson model is fully equivalent to a BCS superconductor and has the same low-energy excitation spectrum, but it is more general: it also describes the transition to a Bose-Einstein condensate for strong pairing and it remains applicable for a very small number of levels. Without the impurity, the Richardson model can be expressed in terms of hard-core bosons (paired electrons) and exactly solved via Bethe ansatz (Richardson-Gaudin equations) [36-40]. The impurity breaks integrability by splitting the electron pairs through exchange scattering, thereby precluding this approach. The problem also cannot be solved using conventional impurity solvers because the bath is interacting, while the mean-field decoupling of the charging term leads to incorrect results [41]. Furthermore, the charge-counting trick [42, 43] is not applicable to a gapped spectrum [41]. A theoretical tool for this family of problems has therefore been sorely lacking and some key questions remained unanswered, in particular whether any states remain present in the gap for odd occupancy of the SC and, if so, what is their nature.

Here we show that Richardson-type Hamiltonians with long-range (all-to-all) interactions coupled to an interacting QD admit a compact representation in terms of matrix product operators (MPO) with small $9 \times 9$ matrices and can be efficiently solved without any approximations in all parameter regimes using the density-matrix renormalization group (DMRG) [44-46]. Possible extensions include the capacitive coupling between the QD and the island [47], the spin-orbit coupling in the SC, the case of a QD in the junction between two islands, as well as various multipleQD problems.

In this work, we systematically investigate the subgap excitations of the simplest situation: a single QD coupled to a single $\mathrm{SC}$ island. The qualitative behavior depends on the ratio of $E_{c}$ over the SC gap $\Delta$. For $E_{c} \lesssim \Delta$, the Kondo coupling drives the YSR singlet-doublet transition. For $E_{c} \gtrsim \Delta$, even-odd effects arise from charge quantization: the occupancy of the SC island varies in steps of one electron similar to a QD in the Coulomb blockade (CB) regime. Subgap states are present also for odd occupancy of the SC, but they disperse very differently compared to even occupancy. The cross-over $E_{c} \approx \Delta$ regime shows complex charging patterns and subgap states with unique properties that strongly depend on the parity of the number of electrons in the superconductor. For parameters that are typical of actual devices, the nature of the subgap states is mixed: it is different from the prototypical YSR states (large- $U$ limit, $\left.E_{c}=0\right)$ and Andreev bound states $(U=0$, $E_{c}=0$ ), as well as from the subgap states of QDs coupled to normal-state Coulomb-blockaded reservoirs. 
Model. The Hamiltonian we study in the present work is $H=H_{\mathrm{imp}}+H_{\mathrm{SC}}+H_{\mathrm{hyb}}$ with [24, 27, 34, 48, 49]

$$
\begin{aligned}
H_{\mathrm{imp}} & =\epsilon \hat{n}_{\mathrm{imp}}+U \hat{n}_{\mathrm{imp}, \uparrow} \hat{n}_{\mathrm{imp}, \downarrow} \\
& =(U / 2)\left(\hat{n}_{\mathrm{imp}}-\nu\right)^{2}+\text { const. } \\
H_{\mathrm{SC}} & =\sum_{i, \sigma} \epsilon_{i} c_{i \sigma}^{\dagger} c_{i \sigma}-\alpha d \sum_{i, j} c_{i \uparrow}^{\dagger} c_{i \downarrow}^{\dagger} c_{j \downarrow} c_{j \uparrow}+E_{c}\left(\hat{n}_{\mathrm{sc}}-n_{0}\right)^{2}, \\
H_{\mathrm{hyb}} & =(v / \sqrt{N}) \sum_{i \sigma}\left(c_{i \sigma}^{\dagger} d_{\sigma}+\text { H.c. }\right) .
\end{aligned}
$$

Here $d_{\sigma}$ and $c_{i \sigma}$ are the annihilation operators corresponding to impurity and bath, $\sigma=\uparrow, \downarrow, \hat{n}_{\text {imp }, \sigma}=d_{\sigma}^{\dagger} d_{\sigma}$ and $\hat{n}_{\mathrm{imp}}=\sum_{\sigma} \hat{n}_{\mathrm{imp}, \sigma} . \epsilon$ is the impurity level controlled by the gate voltage applied to the $\mathrm{QD}, U$ the electron-electron repulsion, and $\nu=1 / 2-\epsilon / U$ is the impurity level in units of electron number. The SC has $N$ levels spaced by $d=2 D / N$ where $2 D$ is the bandwidth, the orbital indexes $i$ and $j$ range between 1 and $N$, the dimensionless coupling constant for pairing interaction is $\alpha, \hat{n}_{\mathrm{sc}}=\sum_{i \sigma} c_{i \sigma}^{\dagger} c_{i \sigma}$, and $n_{0}$ is the gate voltage applied to the SC expressed in units of electron number. The hybridisation strength is $\Gamma=\pi \rho v^{2}$, where $\rho=1 / 2 D$ is the normal-state bath density of states. A schematic representation of this Hamiltonian is shown in Fig. 1, top. Most calculations in this work are performed for $N=800$ and $\alpha=0.23$ (magnitude appropriate for $\mathrm{Al}$ grains [24]), with $D=1$ as the energy unit. The corresponding gap in the thermodynamic limit is $\Delta \approx 0.026 \mathrm{D}$ [41]. The interlevel separation is $d=2 D / N=0.0025 \mathrm{D} \approx \Delta / 10$, thus the finite-size corrections to BCS theory [22,-24, 41, 50, 51] are relatively small [41]. Unless specified otherwise, the QD interaction is $U=0.1 \approx 4 \Delta$ which is a typical value for nanowire devices, and $\Gamma=0.1 U$ which corresponds to intermediately strong coupling. In this work we focus on the situation where the QD-SC system is not strictly isolated but in contact with weakly coupled tunneling probes. The ground state (GS) with fixed (integer) total number of electrons $n=n_{\mathrm{gs}}$ is determined by the gate voltages $\nu$ and $n_{0}$. We use $(0),(+1)$ and $(-1)$ as shorthands for the GS and the lowest-energy (subgap) excited states with occupancy $n_{\text {gs }} \pm 1$, respectively.

Results. The evolution from the YSR to CB regime is clearly visible in the charging diagrams in the $\left(\nu, n_{0}\right)$ plane, see Fig. 1. For small $E_{c}$ there is a $2 e$ periodicity along the $n_{0}$ axis with only a weak even-odd modulation of the subgap state energies, while for large $E_{c}$, the system instead shows a clear $1 e$ periodicity. The transition between the two regimes occurs gradually for $E_{c}$ of order $\Delta$, with the charge stability regions deforming from a pattern of vertical stripes into a well-defined honeycomb diagram. With increasing $\Gamma$, the singlet (blue) regions increase in size because the singlet energy decreases with respect to the doublet energy, while the phase boundaries become smoother (less rectangular) and develop a diagonal slant because for large $\Gamma$ each gate voltage influences occupancy in both parts of the system [41].
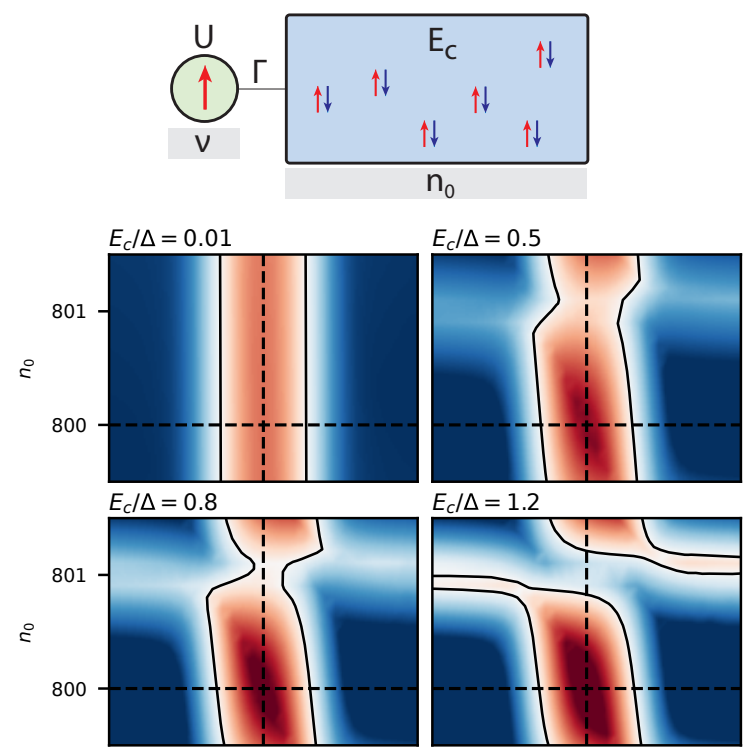

$E_{c} / \Delta=1.2$
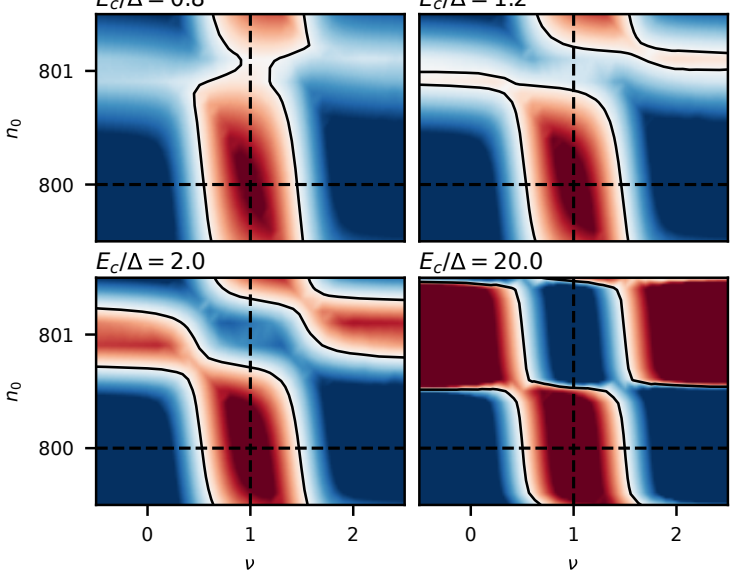

$E_{c} / \Delta=20.0$

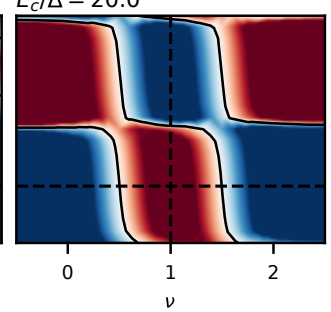

Figure 1. Top: schematic representation of the system. Bottom: phase diagrams as a function of gate voltages applied to the QD $(\nu)$ and to the SC $\left(n_{0}\right)$. Dashed lines correspond to half-filling (particle-hole symmetric) lines of QD and SC at $\nu=1$ and $n_{0}=$ $N=800$. Red: doublet. Blue: singlet. The color indicates the energy difference.

To better understand this evolution, in Fig. 2 we follow the dependence on the coupling $\Gamma$ at fixed $\nu=1$, where the QD hosts a local moment. In the $\Gamma \rightarrow 0$ limit the impurity is decoupled and for $E_{c}<\Delta$ the SC is always in a conventional BCS state with even $n_{\mathrm{sc}}=\left\langle\hat{n}_{\mathrm{sc}}\right\rangle$. For $E_{c} \approx 0$, we uncover the conventional singlet-doublet YSR transition at $T_{K}(\Gamma) / \Delta=0.3$ [7, 12, 52] for a value of $\Gamma$ that does not depend on $n_{0}$; here $T_{K}(\Gamma)$ is the impurity Kondo temperature at the given value of $\Gamma$. With increasing $E_{c}<\Delta$ the transition point moves to larger values of $\Gamma$ around even $n_{0}$ where the charging term makes the existence of Bogoliubov quasiparticles energetically unfavorable. The opposite holds around odd $n_{0}$. As $E_{c}$ grows beyond $\Delta$ we observe a qualitative change. The SC state in the $\Gamma \rightarrow 0$ limit now depends on $n_{0}$ : for $n_{0}$ close to an even integer value, it is a BCS state, while for $n_{0}$ close to an odd integer value, an additional unpaired electron (Bogoliubov quasiparticle) sits at the bottom of the quasiparticle band [19] and for $\Gamma \neq 0$ interacts with the electron at the impurity site via exchange interaction, forming a singlet GS. The exact location of the phase boundary de- 


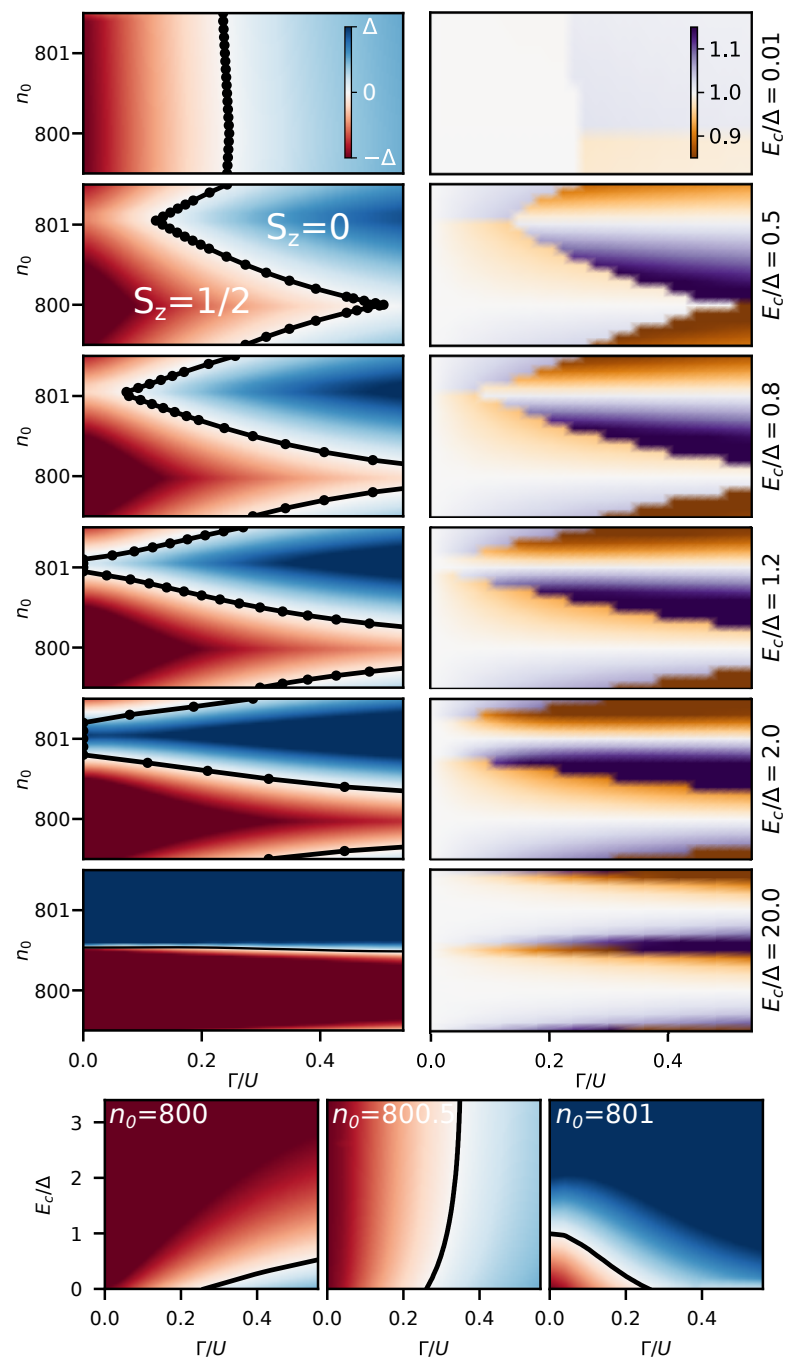

Figure 2. Evolution from the YSR regime to the $\mathrm{CB}$ regime for $\nu=1$. Energy difference $E^{D}-E^{S}$ between the lowest-lying singlet and doublet states in the $\left(\Gamma, n_{0}\right)$ plane for a range of $E_{c}$ (left panels), and in the $\left(\Gamma, E_{c}\right)$ plane for even, half-integer, and odd $n_{0}$ (bottom panels). Red: doublet, blue: singlet, black line: quantum phase transition. Right panels: QD occupancy variation.

pends in a non-trivial way on $\Gamma, U$ and $E_{c}$ due to a threeway competition between Kondo screening, pairing correlations, and Coulomb interaction. The latter also leads to a strong charge redistribution between the QD and SC in the singlet GS, see right panels in Fig. 2. Fig. 3 shows the $E_{c}$ dependence of impurity occupancy, occupancy (charge) fluctuations, and spin correlations at fixed $\Gamma$, for even and odd SC tuning. As $E_{c}$ increases, the charge in the state penalized by the Coulomb term is redistributed, while the charge fluctuations generally decrease, as expected, except for the doublet state in the odd- $n_{0}$ case. The Kondo coupling decreases/increases with increasing $E_{c}$ for even/odd parity of $n_{0}$, which is reflected in the spin-spin correlations of the spin-singlet states.

A striking consequence of the Coulomb repulsion is the

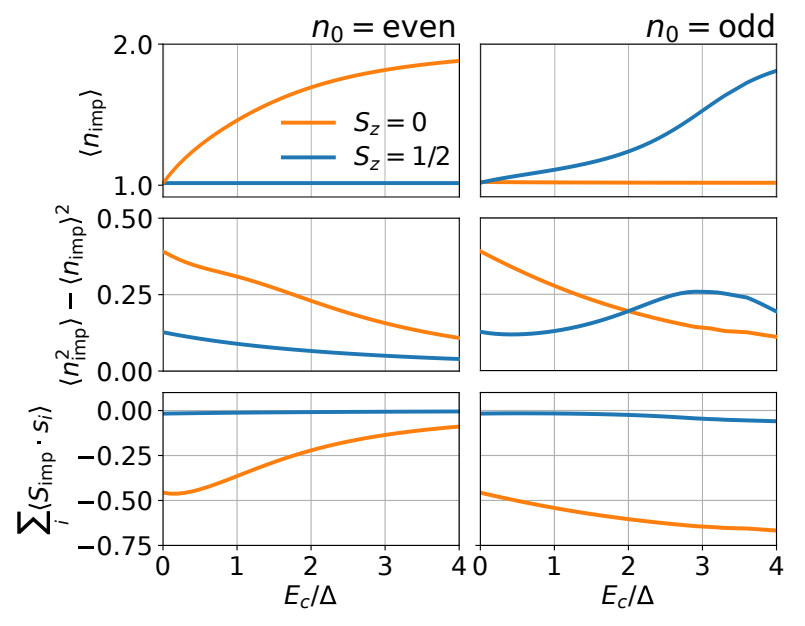

Figure 3. $E_{c}$ dependence of subgap state properties at $\nu=1$.

lack of symmetry in the subgap peak positions except for special points (e.g. $\nu=1$ and even $n_{0}$ ), see Fig. 4. This is a significant departure from the conventional case with $E_{c}=0$, where the peaks are always located exactly at $\omega= \pm E_{\mathrm{YSR}}$, so that the spectra take the form of symmetric eye-shaped loops. For $E_{c} \neq 0$, the states $( \pm 1)$ have in general different excitation energies $E^{ \pm}$leading to drastic changes in the spectral shapes even for small $E_{c}$ (see e.g. black arrows in Fig. 4f). In particular, this leads to discontinuous changes in the spectrum when the total occupancy in the GS changes. For instance, as $\nu$ increases, $E^{+}$decreases until reaching zero, at which point the former $(+1)$ state becomes the new GS. At this point the former $(-1)$ is no longer spectroscopically visible (i.e., it "disappears"), since it has two electrons less than the new GS. The same holds for decreasing $\nu$ for $E^{-}$. An example of such discontinuous changes in the spectrum is indicated by vertical green arrows in Fig. 4b. The spectrum behaves even more remarkably for odd $n_{0}$ (bottom row of Fig. 4). For moderate $E_{c} / \Delta=0.2$, one observes valence skipping (occupancy jump from 800 to 802 , then back to 801 ) due to a redistribution of charge between the $\mathrm{SC}$ and the QD, experimentally visible as a two-sided discontinuity (Fig. 4f, purple arrows), while for $E_{c} \lesssim \Delta$ the excitation are pinched at $\nu=1$. For large $E_{c}>\Delta$, the spectra eventually transform into straight lines typical of CB systems.

Discussion. The nature of the subgap states at $E_{c} \gtrsim \Delta$ is revealed in Fig. 5, where we show the properties of (0), $(+1)$ and $(-1)$ as a function of $\nu$ in panel a), and the $\Gamma$ dependence of the excitations at $\nu=1$ in panel b). We first discuss the case of $\nu=1$. For even $n_{0}$, the GS is a decoupled local-moment state, while the states (+1) and (-1) have impurity occupancies differing by more than half an electron compared to the ground state due to the cost $E_{c}$ of changing the SC occupancy, but they still carry some local moment that aligns antiferromagnetically with respect to SC electrons. The excitations detach from the continuum edge at small $\Gamma$ and shift toward the mid-gap region with increas- 


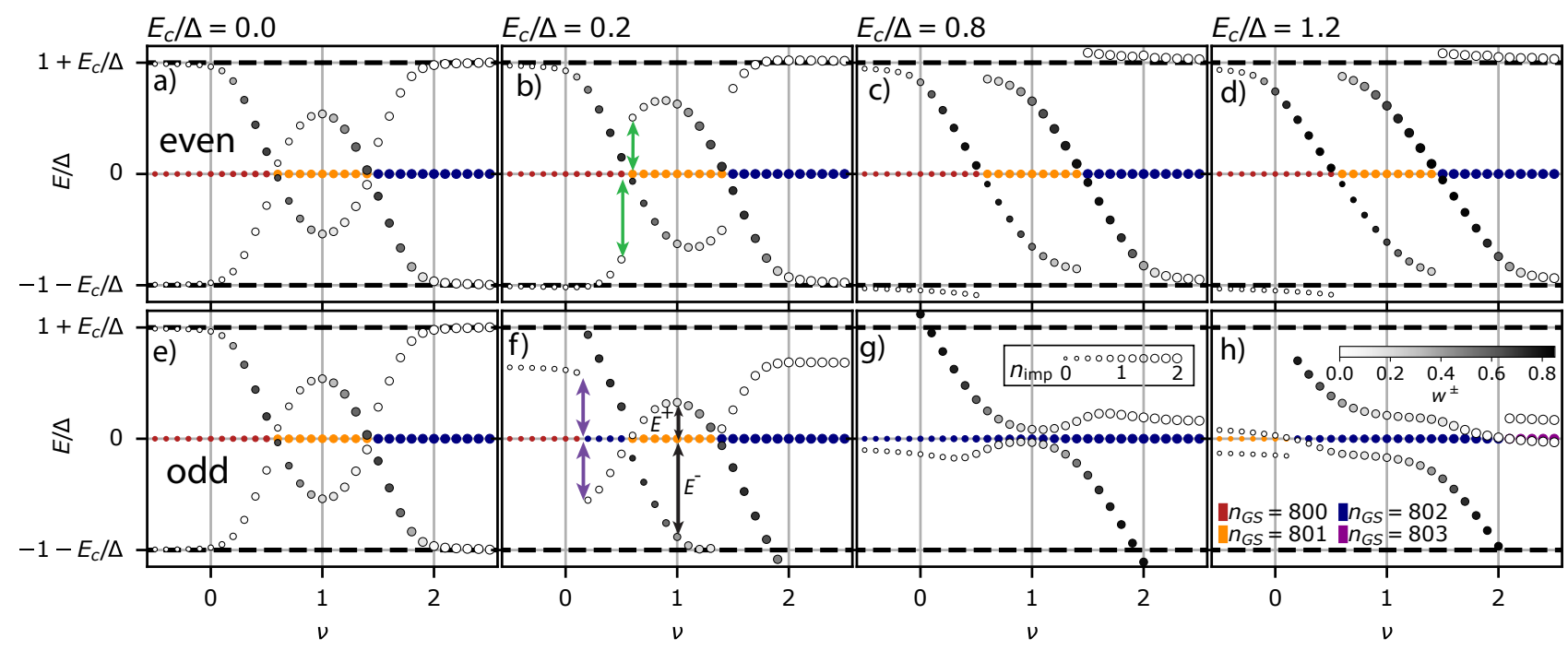

Figure 4. Subgap spectral functions for even (upper row, $n_{0}=800$ ) and odd integer (bottom row, $n_{0}=801$ ) tuning of the superconductor occupancy, as a function of the gate voltage applied on the quantum dot. The positions $E^{+}=E^{(1)}-E^{(0)}$ and $E^{-}=E^{(-1)}-E^{(0)}$ indicate the excitation energies of particle-like (+1) and hole-like (-1) states, the grayscale shows the corresponding spectral weights $w^{ \pm}$(see legend in the inset). The dots at $E=0$ provide information about the ground state: the color encodes the GS charge sector, the size encodes the GS impurity occupancy $n_{\text {imp }}$ (see legend in the inset). The size of grey dots denotes the impurity occupancy in the corresponding excited states. The charge gap for even $n_{0}$ and $\Gamma \rightarrow 0$ is $\Delta+E_{c}$ (dashed lines).

a)

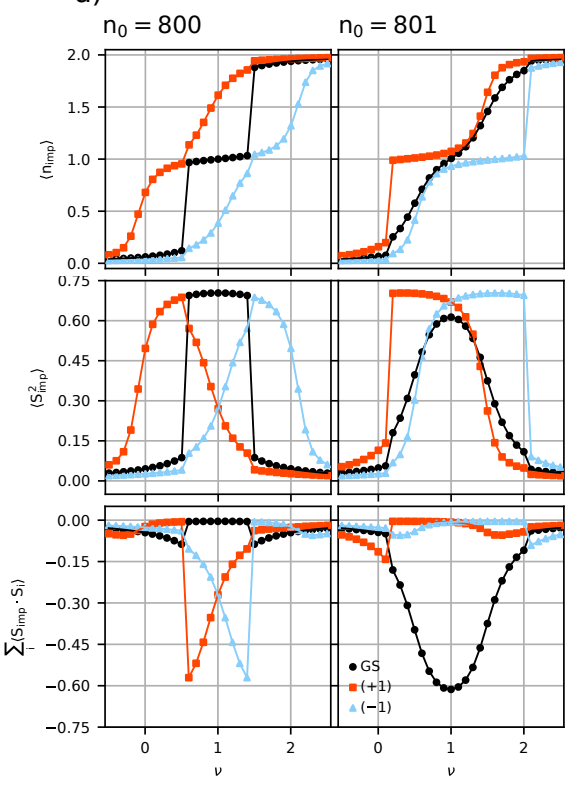

b)

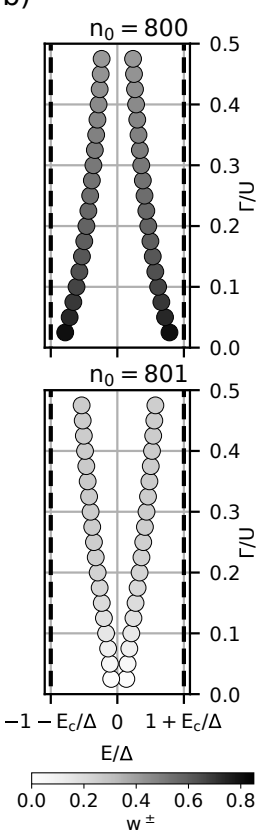

Figure 5. Subgap state properties for $E_{c} / \Delta=1.2$ (right-most panels in Fig. 4). a) Expectation values for $(0),(+1),(-1)$ states vs. gate voltage. b) $\Gamma$ dependence of the subgap spectra at $\nu=1$.

ing $\Gamma$, see Fig. 5 b. The (+1) and (-1) are hence somewhat similar to conventional YSR singlets, although their impurity local-moment is reduced not only through the Kondo mechanism, but also by a very large charge transfer to or

from the superconductor. The excitations may thus be characterized as being YSR-like, sharing some but not all features of the conventional YSR states at $E_{c}=0$. For odd $n_{0}$, the states $(0),(+1),(-1)$ are all similar to each other and carry a local moment at the impurity site. They differ mostly in the presence or absence of the lone Bogoliubov quasiparticle in the SC: (0) has the quasiparticle, while (1) and (+1) do not; (+1) differs from (-1) by the presence of one additional Cooper pair. Adding an electron to the GS costs $E_{c}$ and disrupts the singlet which further costs an energy of order $J_{K} \propto \Gamma$, however at the same time a Cooper pair is formed and the energy $\Delta$ is gained. Indeed, the results for $n_{0}=801$ in panel b) show for small $\Gamma$ approximately linear behavior with a zero intercept at $E_{c}-\Delta=0.2 \Delta$. We thus conclude that for odd $n_{0}$ the subgap states are doublets which result from the disruption of the strong local QD-SC singlet formed by the electron in QD and the lone quasiparticle in SC (see also Fig. S4 for details [41]); these states have no counterparts at all in $E_{c}=0$ systems. Fig. 5 b shows that for even $n_{0}$ the excitations have a large weight on the QD, while the opposite is the case for odd $n_{0}$, where the electron is mostly added to the SC island.

The dispersion ( $\nu$-dependence) of excitations for large $E_{c}$ (e.g. $E_{c} \gtrsim 0.8 \Delta$ ) is strongly affected by the charging terms and it follows the variation of the difference in the impurity occupancy between the ground and excited states, as revealed by comparing Figs. 5 and 4 . For even $n_{0}$ around half filling, the GS occupancy is mostly flat as a function of $\nu$, while the $(+1)$ and (-1) occupancies vary 
rapidly. This is reflected in an equally rapid variation of the excitation spectrum at the same parameters. Along the same line, for odd $n_{0}$ and away from half filling, the occupancy of $(0)$ and $(+1)$ are similar for $\nu>1$, while those of (0) and (-1) are similar for $\nu<1$ and, again, this is reflected in the spectral shape (in this case as flat sections).

Conclusion. Subgap states persist in the presence of large charging energy but they have properties quite unlike YSR states [47]. The proposed method can also address the question of quasiparticle poisoning in Majorana islands [17, 53], gate sensing of charge-tunneling processes [54, 55], superconducting islands on surfaces [5659], topological superconductivity [60], and the existence of Majorana zero modes beyond mean-field [61]. Generalization to multiple bands will find application in multichannel and topological Kondo effects [62-64].

We have benefited from suggestions and discussions with Tadeusz Domansky, Tomáš Novotný, Volker Meden, Marion van Midden, András Pályi, Anton Ramšak, Roman Rausch, and with the members of QDev at the University of Copenhagen. RŽ and LP acknowledge the support of the Slovenian Research Agency (ARRS) under P1-0044. Calculations were performed with the ITensor library [65].

[1] L. Yu, Bound state in superconductors with paramagnetic impurities, Acta Phys. Sin. 21, 75 (1965).

[2] A. I. Rusinov, Superconductivity near a paramagnetic impurity, JETP Lett. 9, 85 (1969), zh. Eksp. Teor. Fiz. Pisma Red. 9, 146 (1968).

[3] H. Shiba, Classical spins in superconductors, Prog. Theor. Phys. 40, 435 (1968).

[4] A. Sakurai, Comments on superconductors with magnetic impurities, Prog. Theor. Phys. 44, 1472 (1970).

[5] S. Nadj-Perge, I. K. Drozdov, J. Li, H. Chen, S. jeon, J. Seo, A. H. MacDonald, B. A. Bernevig, and A. Yazdani, Observation of Majorana fermions in ferromagnetic atomic chains on a superconductor, Science 346, 602 (2014).

[6] J. Zittartz and E. Müller-Hartmann, Theory of magnetic impurities in superconductors. I, Exact solution of the Nagaoka equations, Z. Physik 232, 11 (1970).

[7] K. Satori, H. Shiba, O. Sakai, and Y. Shimizu, Numerical renormalization group study of magnetic impurities in superconductors, J. Phys. Soc. Japan 61, 3239 (1992).

[8] O. Sakai, Y. Shimizu, H. Shiba, and K. Satori, Numerical renormalization group study of magnetic impurities in superconductors. II. Dynamical excitations spectra and spatial variation of the order parameter, J. Phys. Soc. Japan 62, 3181 (1993).

[9] T. Yoshioka and Y. Ohashi, Numerical renormalization group studies on single impurity Anderson model in superconductivity: a unified treatment of magnetic, nonmagnetic impurities, and resonance scattering, J. Phys. Soc. Japan 69, 1812 (2000).

[10] K. G. Wilson, The renormalization group: Critical phenomena and the Kondo problem, Rev. Mod. Phys. 47, 773 (1975).
[11] J.-D. Pillet, P. Joyez, R. Žitko, and M. F. Goffman, Tunneling spectroscopy of a single quantum dot coupled to a superconductor: From Kondo ridge to Andreev bound states, Phys. Rev. B 88, 045101 (2013).

[12] E. J. H. Lee, X. Jiang, R. Žitko, R. Aguado, C. M. Lieber, and S. De Franceschi, Scaling of subgap excitations in a superconductor-semiconductor nanowire quantum dot, Phys. Rev. B 95, 180502(R) (2017).

[13] D. J. Luitz and F. F. Assaad, Weak-coupling continuoustime quantum Monte Carlo study of the single impurity and periodic Anderson models with s-wave superconducting baths, Physical Review B 81, 024509 (2010).

[14] D. J. Luitz, F. F. Assaad, T. Novotný, C. Karrasch, and V. Meden, Understanding the Josephson Current through a Kondo-Correlated Quantum Dot, Physical Review Letters 108, 227001 (2012).

[15] T. Domański, M. Žonda, V. Pokorný, G. Górski, V. Janiš, and T. Novotný, Josephson-phase-controlled interplay between correlation effects and electron pairing in a threeterminal nanostructure, Physical Review B 95, 045104 (2017).

[16] A. Kadlecová, M. Žonda, V. Pokorný, and T. Novotný, Practical guide to quantum phase transitions in quantum-dotbased tunable josephson junctions, Physical Review Applied 11, 044094 (2019).

[17] S. M. Albrecht, E. B. Hansen, A. P. Higginbotham, F. Kuemmeth, T. S. Jespersen, J. Nygård, P. Krogstrup, J. Danon, K. Flensberg, and C. M. Marcus, Transport signatures of quasiparticle poisoning in a Majorana island, Physical Review Letters 118, 137701 (2017).

[18] J. van Veen, A. Proutski, T. Karzig, D. I. Pikulin, R. M. Lutchyn, J. Nygård, P. Krogstrup, A. Geresdi, L. P. Kouwenhoven, and J. D. Watson, Magnetic-field-dependent quasiparticle dynamics of nanowire single-Cooper-pair transistors, Physical Review B 98, 174502 (2018).

[19] D. V. Averin and Y. V. Nazarov, Single-electron charging of a superconducting island, Physical Review Letters 69, 1993 (1992)

[20] B. Jankó, A. Smith, and V. Ambegaokar, BCS superconductivity with fixed number parity, Physical Review B 50, 1152 (1994)

[21] D. Golubev and A. Zaikin, Parity effect and thermodynamics of canonical superconducting ensembles, Physics Letters A 195, 380 (1994).

[22] J. von Delft, A. D. Zaikin, D. S. Golubev, and W. Tichy, Parity-affected superconductivity in ultrasmall metallic grains, Physical Review Letters 77, 3189 (1996).

[23] A. Mastellone, G. Falci, and R. Fazio, Small superconducting grain in the canonical ensemble, Physical Review Letters 80, 4542 (1998).

[24] F. Braun and J. von Delft, Fixed-N superconductivity: The crossover from the bulk to the few-electron limit, Physical Review Letters 81, 4712 (1998)

[25] J. Dukelsky and G. Sierra, Density matrix renormalization group study of ultrasmall superconducting grains, Physical Review Letters 83, 172 (1999).

[26] J. Dukelsky and G. Sierra, Crossover from bulk to fewelectron limit in ultrasmall metallic grains, Physical Review B 61, 12302 (2000)

[27] F. Braun and J. von Delft, Superconductivity in ultrasmall metallic grains, Physical Review B 59, 9527 (1999). 
[28] K. Van Houcke, S. M. A. Rombouts, and L. Pollet, Survival of parity effects in superconducting grains at finite temperature, Physical Review B 73, 132509 (2006).

[29] S. M. A. Rombouts, K. Van Houcke, and L. Pollet, Loop updates for quantum Monte Carlo simulations in the canonical ensemble, Physical Review Letters 96, 180603 (2006).

[30] M. T. Tuominen, J. M. Hergenrother, T. S. Tighe, and M. Tinkham, Experimental evidence for parity-based 2e periodicity in a superconducting single-electron tunneling transistor, Physical Review Letters 69, 1997 (1992).

[31] P. Lafarge, P. Joyez, D. Esteve, C. Urbina, and M. H. Devoret, Two-electron quantization of the charge on a superconductor, Nature 365, 422 (1993).

[32] P. Lafarge, P. Joyez, D. Esteve, C. Urbina, and M. H. Devoret, Measurement of the even-odd free-energy difference of an isolated superconductor, Physical Review Letters 70, 994 (1993).

[33] T. M. Eiles, J. M. Martinis, and M. H. Devoret, Even-odd asymmetry of a superconductor revealed by the Coulomb blockade of Andreev reflection, Physical Review Letters 70, 1862 (1993)

[34] J. von Delft and D. Ralph, Spectroscopy of discrete energy levels in ultrasmall metallic grains, Physics Reports 345, 61 (2001)

[35] P. W. Anderson, New approach to the theory of superexchange interactions, Phys. Rev. 115, 2 (1959).

[36] R. Richardson, A restricted class of exact eigenstates of the pairing-force Hamiltonian, Physics Letters 3, 277 (1963)

[37] R. Richardson and N. Sherman, Exact eigenstates of the pairing-force Hamiltonian, Nuclear Physics 52, 221 (1964).

[38] R. W. Richardson, Numerical study of the 8-32-particle eigenstates of the pairing Hamiltonian, Physical Review 141, 949 (1966)

[39] J. von Delft and F. Braun, Superconductivity in ultrasmall grains: Introduction to Richardson's exact solution, condmat/9911058 (1999).

[40] G. Sierra, J. Dukelsky, G. G. Dussel, J. von Delft, and F. Braun, Exact study of the effect of level statistics in ultrasmall superconducting grains, Physical Review B 61, R11890 (2000)

[41] Supplementary material contains additional results, detailed model definition, discussion of excitation energies in superconducting islands, MPO representation of the Hamiltonian, and the results of benchmark calculations for method validation.

[42] E. Lebanon, A. Schiller, and F. B. Anders, Coulomb blockade in quantum boxes, Physical Review B 68, 041311(R) (2003).

[43] F. B. Anders, E. Lebanon, and A. Schiller, Coulomb blockade and non-Fermi-liquid behavior in quantum dots, Phys. Rev. B 70, 201306(R) (2004).

[44] S. R. White, Density matrix formulation for quantum renormalization groups, Phys. Rev. Lett. 69, 2863 (1992).

[45] U. Schollwöck, The density-matrix renormalization group in the age of matrix product states, Annals of Physics 326, 96 (2011), january 2011 Special Issue.

[46] G. M. Crosswhite, A. C. Doherty, and G. Vidal, Applying matrix product operators to model systems with long-range interactions, Phys. Rev. B 78, 035116 (2008).

[47] J. C. E. Saldaña, A. Vekris, L. Pavešić, P. Krogstrup, R. Žitko, K. Grove-Rasmussen, and J. Nygård, Bias asymmetric subgap states mimicking Majorana signatures, arXiv:2101.10794 (2021).

[48] P. W. Anderson, Localized magnetic states in metals, Phys. Rev. 124, 41 (1961).

[49] J. Dukelsky, S. Pittel, and G. Sierra, Colloquium: Exactly solvable Richardson-Gaudin models for many-body quantum systems, Reviews of Modern Physics 76, 643 (2004).

[50] J. M. Román, G. Sierra, and J. Dukelsky, Elementary excitations of the BCS model in the canonical ensemble, Physical Review B 67, 064510 (2003).

[51] E. A. Yuzbashyan, A. A. Baytin, and B. L. Altshuler, Strong-coupling expansion for the pairing Hamiltonian for small superconducting metallic grains, Physical Review B 68, 214509 (2003).

[52] R. Zitko, J. S. Lim, R. Lopez, and R. Aguado, Shiba states and zero-bias anomalies in the hybrid normalsuperconductor Anderson model, Phys. Rev. B 91, 045441 (2015).

[53] R. M. Lutchyn, E. P. A. M. Bakkers, L. P. Kouwenhoven, P. Krogstrup, C. M. Marcus, and Y. Oreg, Majorana zero modes in superconductor-semiconductor heterostructures, Nature Reviews Materials 3, 52 (2018).

[54] J. van Veen, D. de Jong, L. Han, C. Prosko, P. Krogstrup, J. D. Watson, L. P. Kouwenhoven, and W. Pfaff, Revealing charge-tunneling processes between a quantum dot and a superconducting island through gate sensing, Physical Review B 100, 174508 (2019).

[55] D. de Jong, J. van Veen, L. Binci, A. Singh, P. Krogstrup, L. P. Kouwenhoven, W. Pfaff, and J. D. Watson, Rapid detection of coherent tunneling in an InAs nanowire quantum dot through dispersive gate sensing, Physical Review Applied 11, 044061 (2019).

[56] S. Vlaic, S. Pons, T. Zhang, A. Assouline, A. Zimmers, C. David, G. Rodary, J.-C. Girard, D. Roditchev, and H. Aubin, Superconducting parity effect across the Anderson limit, Nature Communications 8, 14549 (2017).

[57] G. C. Ménard, S. Guissart, C. Brun, R. T. Leriche, M. Trif, F. Debontridder, D. Demaille, D. Roditchev, P. Simon, and T. Cren, Two-dimensional topological superconductivity in $\mathrm{Pb} / \mathrm{Co} / \mathrm{Si}(111)$, Nature Communications 8, 2040 (2017).

[58] T. Zhang, S. Vlaic, S. Pons, A. Assouline, A. Zimmers, D. Roditchev, H. Aubin, G. Allan, C. Delerue, C. David, G. Rodary, and J.-C. Girard, Quantum confinement effects in $\mathrm{Pb}$ nanocrystals grown on InAs, Physical Review B 97, 214514 (2018).

[59] A. Palacio-Morales, E. Mascot, S. Cocklin, H. Kim, S. Rachel, D. K. Morr, and R. Wiesendanger, Atomicscale interface engineering of majorana edge modes in a $2 d$ magnet-superconductor hybrid system, Science Advances 5, eaav6600 (2019)

[60] M. F. Lapa and M. Levin, Rigorous results on topological superconductivity with particle number conservation, Physical Review Letters 124, 257002 (2020).

[61] G. Ortiz, J. Dukelsky, E. Cobanera, C. Esebbag, and C. Beenakker, Many-body characterization of particleconserving topological superfluids, Physical Review Letters 113, 267002 (2014).

[62] R. Žitko and M. Fabrizio, Non-Fermi-liquid behavior in quantum impurity models with superconducting channels, Phys. Rev. B 95, 085121 (2017).

[63] B. Béri and N. R. Cooper, Topological Kondo effect with 
Majorana fermions, Physical Review Letters 109, 156803 (2012).

[64] M. Papaj, Z. Zhu, and L. Fu, Multichannel charge Kondo effect and non-Fermi-liquid fixed points in conventional and topological superconductor islands, Physical Review B 99, 014512 (2019).

[65] ITensor Library (version 2.0.11) http://itensor.org.

[66] A more precise statement is that the mean-field approximation is appropriate in the limits $E_{c} \ll U$ and $E_{c} \gg U$, where either of the two charge repulsion terms fixes the occupancies in both parts of the system.

[67] K. A. Matveev, Quantum fluctuations of the charge of a metal particle under the Coulomb blockade conditions, $\mathrm{Zh}$. Eksp. Theor. Fiz. 99, 1598 (1991), Sov. Phys. JETP 72, 892 (1991).

[68] H. Schoeller and G. Schön, Mesoscopic quantum transport: Resonant tunneling in the presence of a strong Coulomb interaction, Physical Review B 50, 18436 (1994)

[69] A. C. Hewson, The Kondo Problem to Heavy-Fermions (Cambridge University Press, Cambridge, 1993).

[70] R. Bulla, T. A. Costi, and T. Pruschke, The numerical renormalization group method for quantum impurity systems, Rev. Mod. Phys. 80, 395 (2008).

[71] F. A. Wolf, I. P. McCulloch, and U. Schollwöck, Solving nonequilibrium dynamical mean-field theory using matrix product states, Physical Review B 90, 235131 (2014).

[72] R. Žitko and T. Pruschke, Energy resolution and discretization artefacts in the numerical renormalization group, Phys. Rev. B 79, 085106 (2009).

[73] R. Žitko, Quantitative determination of the discretization and truncation errors in numerical renormalization-group calculations of spectral functions, Phys. Rev. B 84, 085142 (2011). 


\section{SUPPLEMENTAL MATERIAL}

Here we discuss the failure modes of alternative approaches for solving the impurity problems with superconducting bath and Coulomb blockade (mean-field decoupling and charge-counting auxiliary operator approach), show a number of additional results that supplement those presented in the main text, provide the details on the method (including a detailed account of the Hamiltonian and its matrix-product-operator representation), and present some results of benchmark calculations for method validation.

\section{Failure of the mean-field decoupling of the charging term}

The Hamiltonian discussed in this work contains interaction terms of three types: 1) pairing interaction in the superconductor, 2) on-site charge repulsion on the impurity site, 3) charge repulsion in the superconducting island. This raises the question of possible simplifications of the problem through mean-field decoupling of certain terms. For problem sizes (number of levels in the SC island) considered in this work, the mean-field decoupling of the pairing interaction to the BCS mean-field form $\Delta c_{i \uparrow}^{\dagger} c_{i \downarrow}^{\dagger}$ is a perfectly valid approximation. In our scheme, we do not perform this step for purely practical reasons: it does not simplify the calculations (in fact, the loss of the the total charge as a conserved quantum number would only make them more difficult from the numerical perspective). The meanfield decoupling on the impurity on-site repulsion is well known to lead to qualitatively incorrect results (e.g., the well-known unphysical spontaneous spin symmetry breaking in the unrestricted Hartree-Fock solution of the Anderson impurity model for $U>\pi \Gamma$ [48]). The remaining question is thus that of the $E_{c}\left(\hat{n}_{\mathrm{sc}}-n_{0}\right)^{2}$ term in the superconducting bath, whose mean-field decoupling might at first seem innocuous. If this term could be safely decoupled without affecting the qualitative nature of the subgap states and without severe quantitative issues, this would enable the use of traditional quantum impurity solvers, such as NRG and CTQMC.

We thus look into this question more closely and consider two approximative schemes. One consists in the traditional mean-field decoupling of this term (the "mean-field" scheme), leading to the following quadratic form:

$$
H^{\prime}=\left(\hat{n}_{\mathrm{sc}}-n_{0}\right)^{2} \approx 2 \hat{n}_{\mathrm{sc}}\left(\left\langle\hat{n}_{\mathrm{sc}}\right\rangle-n_{0}\right)+n_{0}^{2}-\left\langle\hat{n}_{\mathrm{sc}}\right\rangle^{2} .
$$

The problem then needs to be solved self-consistently, for each state separately (we note that using ground-state expectation values for all states leads to unphysical discontinuities across the quantum phase transitions).

The other scheme (the "static" scheme) is a more severe approximation. It consists in performing the calculations for $E_{c}=0$, then simply shifting the resulting energy by $E_{c}\left(\left\langle\hat{n}_{\mathrm{sc}}\right\rangle-n_{0}\right)^{2}$, again for each state separately.
We note that both the "mean-field" and the "static" schemes fully neglect the correlation effects of the $E_{c}$ term and retain only its average effect, but they depend in the implementation details: the first is self-consistent and incorporates the static effect of the $E_{c}$ term on the wavefunction, while the second is a one-shot calculation at $E_{c}=0$, where the wavefunction does not depend on $E_{c}$ and $n_{0}$ at all, only the energy is shifted.

For purposes of comparing these approaches, we performed all calculations using the same DMRG impurity solver, the only difference being the different level of the approximation in the charging term. The $E_{c}$ dependence at even $n_{0}$ tuning are shown in Fig. S1 (a). We consider two cases, that of large $U / \Delta=30$ where the QD behaves as a Kondo impurity to a good approximation, and that of $U / \Delta=4$ which is appropriate for real devices. We show two energies. The energy $E$ shown in the first row is the energy as evaluated within the approximation scheme: this is the energy that the different methods produce as their final answer. The expectation value $\langle H\rangle$ in the second row is computed using the wavefunctions from the different schemes: this is the actual energy of the approximate wavefunctions, which can be used as a gauge for their quality (the exact DMRG solution is the absolute ground state of the problem with minimal energy).

For $U / \Delta=30$, the differences are only quantitative and relatively weak. This is expected, since large $U$ enforces small charge fluctuations on the impurity site, thereby reducing the effects of the charging term in the range of $E_{c}$ values of interest (order $\Delta$ ). We find that the discrepancy is larger for the singlet state, where the "static" method seemingly performs slightly better than the self-consistent "mean-field" method. For the doublet state, both approximate schemes produce the same result. This is due to the symmetry of the problem: the system is particle-hole symmetric in this case, with $\left\langle\hat{n}_{\mathrm{sc}}\right\rangle=n_{0}$. The reason for the energy difference with respect to the exact solution is the charge fluctuations, i.e., the difference between $\left\langle\left(\hat{n}_{\mathrm{sc}}-n_{0}\right)^{2}\right\rangle$ and $\left(\left\langle\hat{n}_{\mathrm{sc}}\right\rangle-n_{0}\right)^{2}$, which is non-zero even in the presence of particle-hole symmetry. In addition, the inexact treatment of charge fluctuations on the SC island also affects the results for charge fluctuations on the impurity site (third row): the decrease of the impurity charge fluctuations in the doublet state with increasing $E_{c}$ is not captured at all at the mean-field level, while the fluctuations in the singlet state grow rather than slightly decrease. The plot of $\langle H\rangle$ (second row) shows that the error quantified by the difference in energy expectation values is small for the doublet states, but more significant for the singlet states. Interestingly, the differences in $E$ are significantly larger than those in $\langle H\rangle$ for the "static" method, while the "mean-field" method shows the opposite. This implies that the differences in $E$ (i.e., the measurable excitation energies, shown in the fourth row) are quite small for the "static" method, while the discrepancy is somewhat larger for the "mean-field" method. In spite of all these quanti- 
a)

a) $U / \Delta=30$
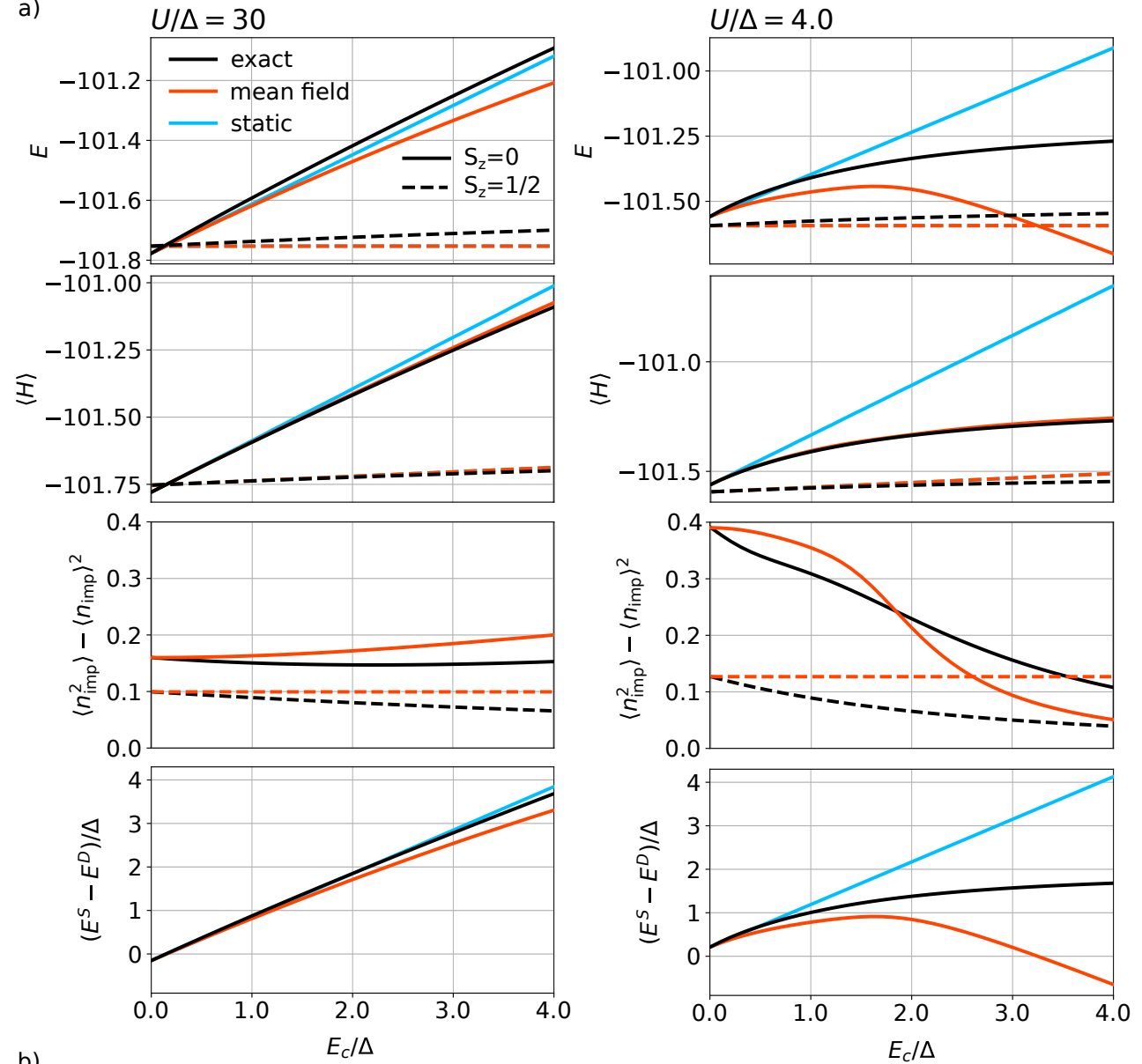

b)
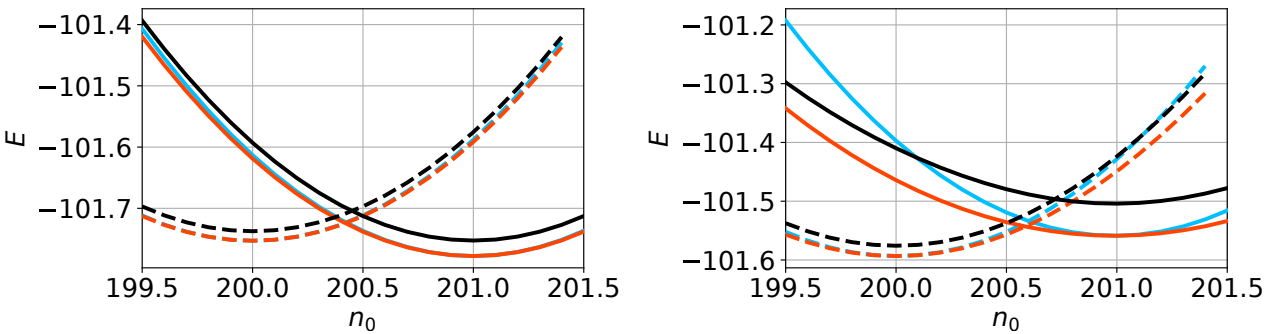

Figure S1. Comparison of the mean-field decoupling schemes for large $U / \Delta$ (Kondo limit) and moderate $U / \Delta$ (realistic value for actual devices). We compare exact results from the DMRG solution (full lines) with the results of two approximate schemes (dashes lines). (a) $E_{c}$ dependence of energy at fixed $n_{0}=200$. First row: energies obtained by the different approaches (see text for details). Second row: expectation value of the full Hamiltonian evaluated with the wavefunction obtained in the different approaches. Third row: charge fluctuations. Fourth row: excitation energy. (b) $n_{0}$ dependence of energy at fixed $E_{c}=X X$. Parameters are $\Gamma / U=0.2$, $\alpha=0.4, N=200$.

tative differences, we conclude that at this large value of $U / \Delta=30$, the charging term in the superconductor is for the most part adequately captured by the mean-field approximations (which will in fact be used in the following when discussing the large- $U$ limit, see Eq. (4) in the section on phase diagrams of the Supplemental Material). For such large $U / \Delta$ values, the states thus still have the nature of the standard YSR states even at large $E_{c} / \Delta$, only with shifted energies.

At realistic $U / \Delta=4$, the discrepancies are more se- vere (see the right-hand panels). This is especially the case when evaluating the energies $E$ within the approximate schemes, rather than evaluating the expectation value of the original Hamiltonian, $\langle\hat{H}\rangle$. This holds in particular for the singlet states, which arise from a strong coupling between the QD and SC electrons. The energies of the three aproaches start strongly diverging already at low $E_{c} / \Delta$ (order 0.1 ), while at $E_{c} / \Delta \approx 1$ the error is sufficiently large that the results are highly questionable. The mean-field approach fails even qualitatively, predicting a 
quantum phase transition which does not really exist. At the same time, the mean-field wavefunction appears to be reasonably good as far as its energy expectation value is concerned. The plots of charge fluctuations reveal that the mean-field methods nevertheless poorly describe the dynamical aspects of the problem. We thus conclude that at the experimentally relevant values only the full solution leads to acceptable results.

The dependence of energies on the gate voltage $n_{0}$ at fixed $E_{c}$ are shown in Fig. S1 (b). We again find that the approximations are adequare in the limit of large $U / \Delta$, while for realistic $U / \Delta=4$ the deviations become sizable. In particular, we note that the curvatures of the singletstate parabolas (centered at $n_{0} \approx 201$ ) are different. The "static" method does not capture the renormalization of $E_{c}$. The "mean-field" method does, but at the price of a sizable systematic error that is constant in $n_{0}$.

We conclude that the treatment of the charging term at the mean-field level is sufficient to obtain some basic understanding of the overall trends in the limits of low $E_{c}$ [66], but it does not capture the changing nature of the subgap states (evolution from conventional YSR towards subgap states with Coulombic nature, characterized by local moment reduction via charge redistribution), produces spurious qualitative features (phase transitions which do not actually exist), and is insufficiently accurate at quantitative level to be of real use in the interpretation of experiments.

\section{Failure of the charge-counting trick}

Impurity models with a Coulomb term $E_{c} \hat{n}^{2}$ can be mapped on an effective model using collective charge operators [67, 68] and solved using the numerical renormalization group (NRG) techniques [42, 43]. The idea is to replace the hopping term

$$
\sum_{i \sigma} c_{i \sigma}^{\dagger} d_{\sigma}+\text { H.c. }
$$

by

$$
\sum_{i \sigma} \hat{N}^{+} c_{i \sigma}^{\dagger} d_{\sigma}+\text { H.c. }
$$

after introducing collective charge operators for electrons on the island, $\hat{N}=\sum_{m=-\infty}^{\infty} m|m\rangle\langle m|, \hat{N}^{ \pm}=$ $\sum_{m=-\infty}^{\infty}|m \pm 1\rangle\langle m|$, converting $E_{c}\left(\hat{n}_{\mathrm{sc}}-n_{0}\right)^{2}$ to $E_{c}(\hat{N}-$ $\left.n_{0}\right)^{2}$, finally relaxing the constraint $\hat{N}=\hat{n}_{\text {sc }}$ and thus regarding $\hat{N}$ as an independent degree of freedom. This approach is applicable when the dynamics of the collective charge operator is insensitive to the precise number of conduction electrons in the bands [42]. This is the case for normal-state bands, but not for gapped systems such as superconductors, where this approach leads to incorrect excitation spectra. We find that the spectra contain not only additional spurious states that would need to be projected out (replicas of physical states at higher energies which do not meet the constraint), but even the expected physical excitations have incorrect energies. This is a fundamental issue that does not appear to have a practical solution. For this reason, it appears unlikely that conventional impurity solvers will ever be adapted to problems with gapped continuum in the presence of a Coulomb interaction term.

\section{Finite-size effects}

Ultra-small superconducting islands have excitation spectra which significantly differ from the BCS spectra in the large- $N$ limit, with some elementary excitations which have no counterpart in the Bogoliubov picture [50, 51]. We assess the effect of the ratio between the interlevel separation $d=2 D / N$ and the BCS gap $\Delta$ on the Yu-ShibaRusinov states of the DMRG solution of the QD-SC problem in Fig. S2 When scaled in terms of the superconducting gap of the finite-size system (obtained as the $\Gamma \rightarrow 0$ limit of the YSR excitation energy), the curves tend to approach the asymptotic YSR curve from above or below for odd and even $N$, respectively. For $N=800$, used in most calculations in this work, the Bogoliubov picture is valid and the results are even quantitatively close to those for a superconductor in the thermodynamic limit, although some finite-size corrections to the BCS mean-field theory remain present. The superconducting islands in the contemporary hybrid devices are sufficiently large that the effects beyond the BCS theory need not be considered.

\section{Phase diagrams}

Fig. S3 presents the phase diagrams at fixed impurity gate voltage $\nu=1$ for several values of $U$ to supplement those for $U=0.1 \approx 4 \Delta$ shown in Fig. 1 of the main text.

For $U=10 \gg D, E_{c}, \Delta$ and $\nu=1$, the QD occupancy is pinned to $n_{\mathrm{imp}} \approx 1$; the QD is then a pure exchange scatterer with the Kondo exchange coupling constant $J_{K}=8 \Gamma / \rho \pi U$. In this case, the phase diagram can be well reproduced using the following approximation to the many-body energy levels in even/odd $n_{\text {sc }}$ sectors:

$E\left(n_{\mathrm{sc}}\right) \approx \mathrm{const}+E_{c}\left(n_{\mathrm{sc}}-n_{0}\right)^{2}+ \begin{cases}0 & n_{\mathrm{sc}} \text { even }, \\ \Delta-E_{B}(\Gamma) & n_{\mathrm{sc}} \text { odd },\end{cases}$

where $E_{B}(\Gamma)$ is the binding energy of the YSR quasiparticle, such that $\lim _{\Gamma \rightarrow 0} E_{B}(\Gamma)=0$. The data for $E_{B}(\Gamma)$ can be taken e.g. from a NRG calculation. This is the idealized version of Fig. 1 from main text, showing qualitatively similar evolution for YSR to CB regimes, but with large quantitative differences, in particular in the crossover $E_{c} \approx \Delta$ regime where the competition between various physical mechanisms is the most pronounced. In the large- $U$ limit, at half-integer filling of the superconductor 

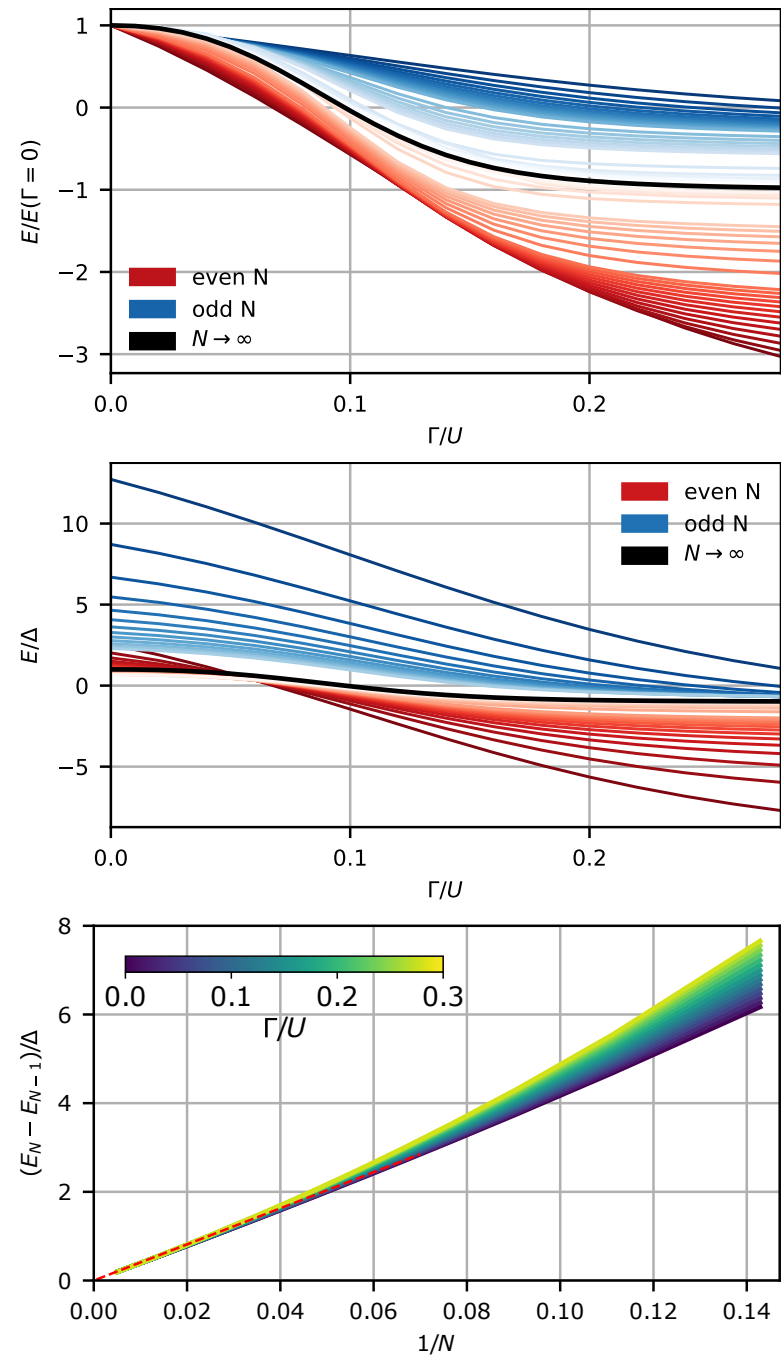

Figure S2. Finite-size effects in the YSR excitation spectra. Top: YSR energies normalized by the superconducting gap at given $N$ (equal to the $\Gamma \rightarrow 0$ limit of the subgap excitation energy). We plot all $N$ from 6 to 30 , then pairs $(40,41),(50,51), \ldots(90$, $91)$, then pairs $(100,101),(200,201), \ldots,(600,601)$. Middle: YSR energies normalized by the BCS gap obtained in the large$N$ limit. We plot all $N$ from 6 to 30 . Bottom: $d \propto 1 / N$ scaling of the difference between even $N$ and odd $N$ results for a range of $\Gamma / U$ ratios. The asymptotic large- $N$ behavior of the difference is linear in $1 / N$ (red dashed line). Here $U=10$.

$\left(n_{0}=800.5\right)$ the transition between singlet and doublet GS occurs at essentially the same value of $\Gamma$ for all values of $E_{c}$ due to equal charging energies for both states, as follows from Eq. (4), because for large $U$ the only dependence on $n_{0}$ is explicitly through the charging term: the transition then occurs for $E_{B}(\Gamma)=\Delta$.

Eq. (4) also explains the change of topology in the phase diagrams at $E_{c}=\Delta$. This occurs because the binding energy of the YSR quasiparticles is bounded as $0<$ $E_{B}(\Gamma)<2 \Delta$. For $E_{c}>\Delta$ it is no longer possible to trap a Bogoliubov quasiparticle at the impurity site for even $n_{0}$, thus the system remains in a doublet ground state for any value of $\Gamma$.

For $U=1$, the system is essentially still in the deep Kondo regime. The quantitative differences at $U=0.1$ (the value corresponding to all results shown in the main text) are, however, significant. They are most apparent in panel b) showing the phase diagram in the $\left(\Gamma, E_{c}\right)$ plane. For even $n_{0}=800$, we observe a significantly slower approach to the $E_{c}=\Delta$ asymptote compared to the large- $U$ limit. For half-integer $n_{0}=800.5$ the quantum phase transition value of hybridisation $\left(\Gamma_{C}\right)$ exhibits a weak $E_{c}$ dependence, thus the transition line is no longer strictly vertical. This is due to the competition between the $E_{c}$ and $U$ terms, i.e., due to the redistribution of charge which is now possible even at the half-way $n_{0}=800.5$ point because of the weaker electron-electron repulsion $U$ on the QD. The diagram for odd $n_{0}=801$ appears to be less affected.

Finally, for very low $U=0.01$ the system is in a qualitatively different weak-interaction regime where the subgap states are better described as Andreev bound states (ABS). Here the increasing $E_{c} / \Delta$ ratio drives a cross-over between the $\mathrm{ABS}$ and $\mathrm{CB}$ regimes that still shows some similarities with the YSR-CB cross-over. The discussion of this regime is beyond the scope of this work.

\section{Charging diagrams}

In Fig. S4a we show additional charging diagrams to supplement those shown in Fig. 2 of the main text. Increasing $\Gamma$ leads to more diffuse appearance of the charging patterns and more dominant diagonal striping, which is a consequence of the formation of a "large single quantum dot" comprising both the original QD and the SC island with the effective level controlled by the sum of gate voltages, $n_{0}+\nu$. As an aid in the interpretation of these diagrams, in Figs. $\mathbf{S 4} \mathrm{b}$ and $\mathrm{S} 4 \mathrm{c}$ we show the impurity occupancy using two representations: as density plot that can be directly compared with the charging diagrams, and additionally as line cuts at even $n_{0}=800$, half-integer $n_{0}=800.5$ and odd $n_{0}=801$. With increasing $\Gamma$ the variation of $\left\langle n_{\text {imp }}\right\rangle$ with $\nu$ becomes increasingly smooth and the local magnetic moment for $\nu \approx 1$ becomes less defined (for $\Gamma / U=0.5$ one has $U / \pi \Gamma \approx 0.64<1$, hence no local moment in the Hartree-Fock picture [48]). This effect is partly compensated by increasing $E_{c}$ which reduces the charge fluctuations betweed the QD and the $\mathrm{SC}$ island. For instance, the local moment reemerges in the case of $\Gamma / U=0.5$ for $E_{c} / \Delta \gtrsim 0.8$ when $n_{0}$ is even. This is concomitant with the appearance of a doublet region in the phase diagram. For odd $n_{0}$ this process is less efficient, QD occupancy becomes quantized only for very large values of $E_{c}$. 

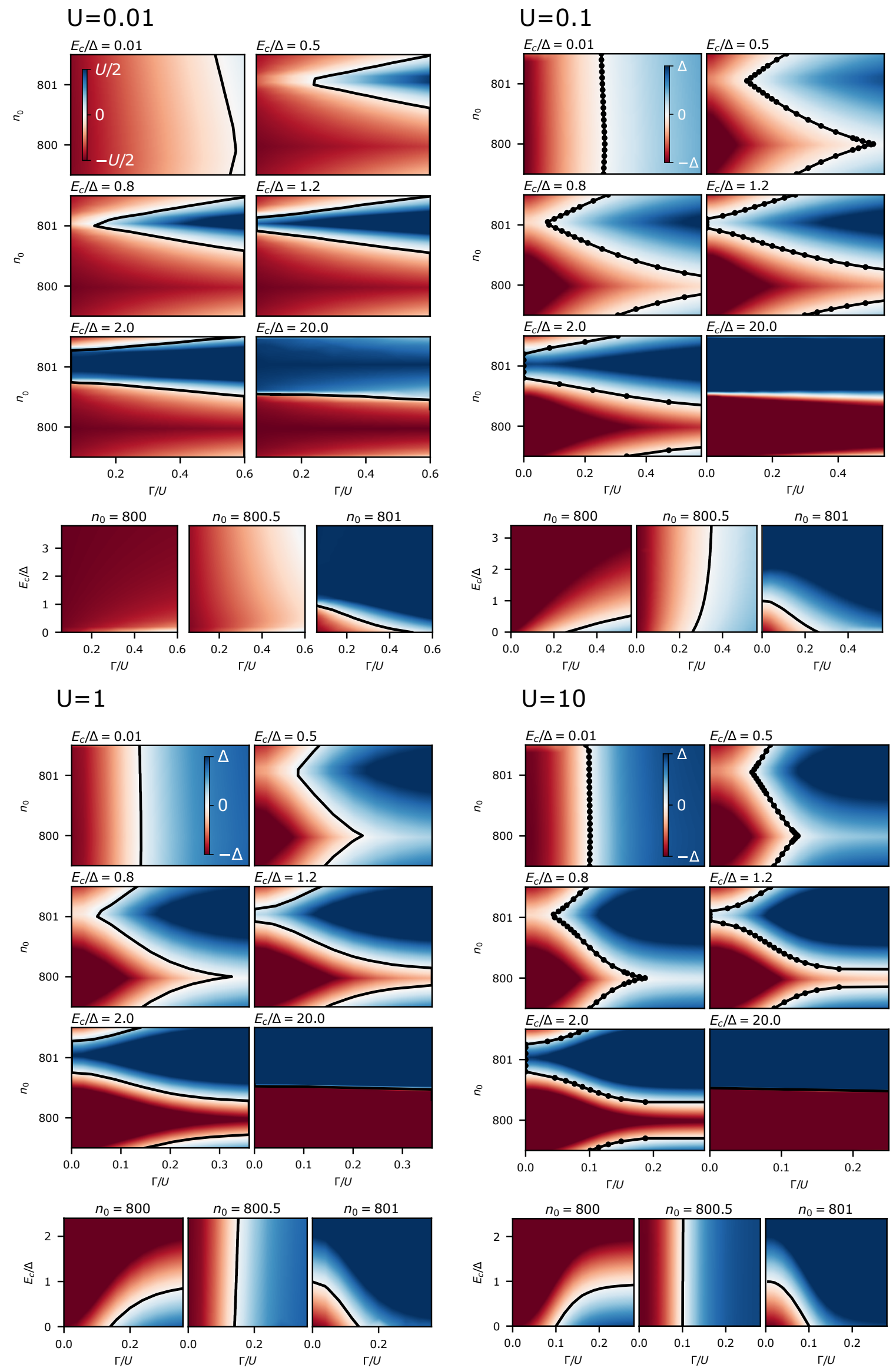

Figure S3. Quantum phase transition between the doublet and singlet phases for different values of $U$. The case of $U=0.1$ is shown in Fig. 1 of the main text. 
a)

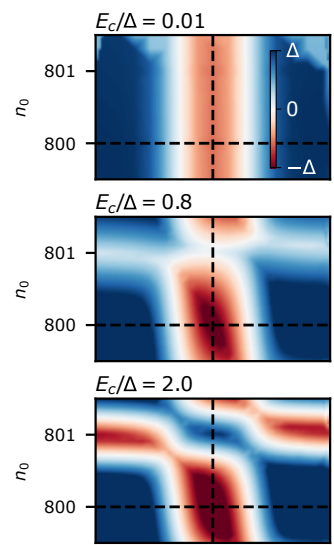

b)
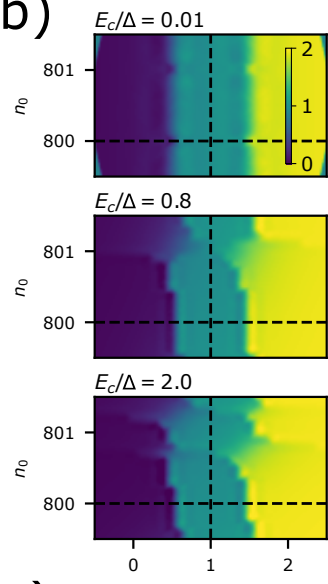

C) $n_{0}=800.0$

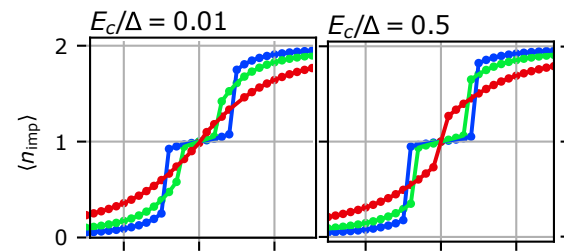

$n_{0}=800.5$
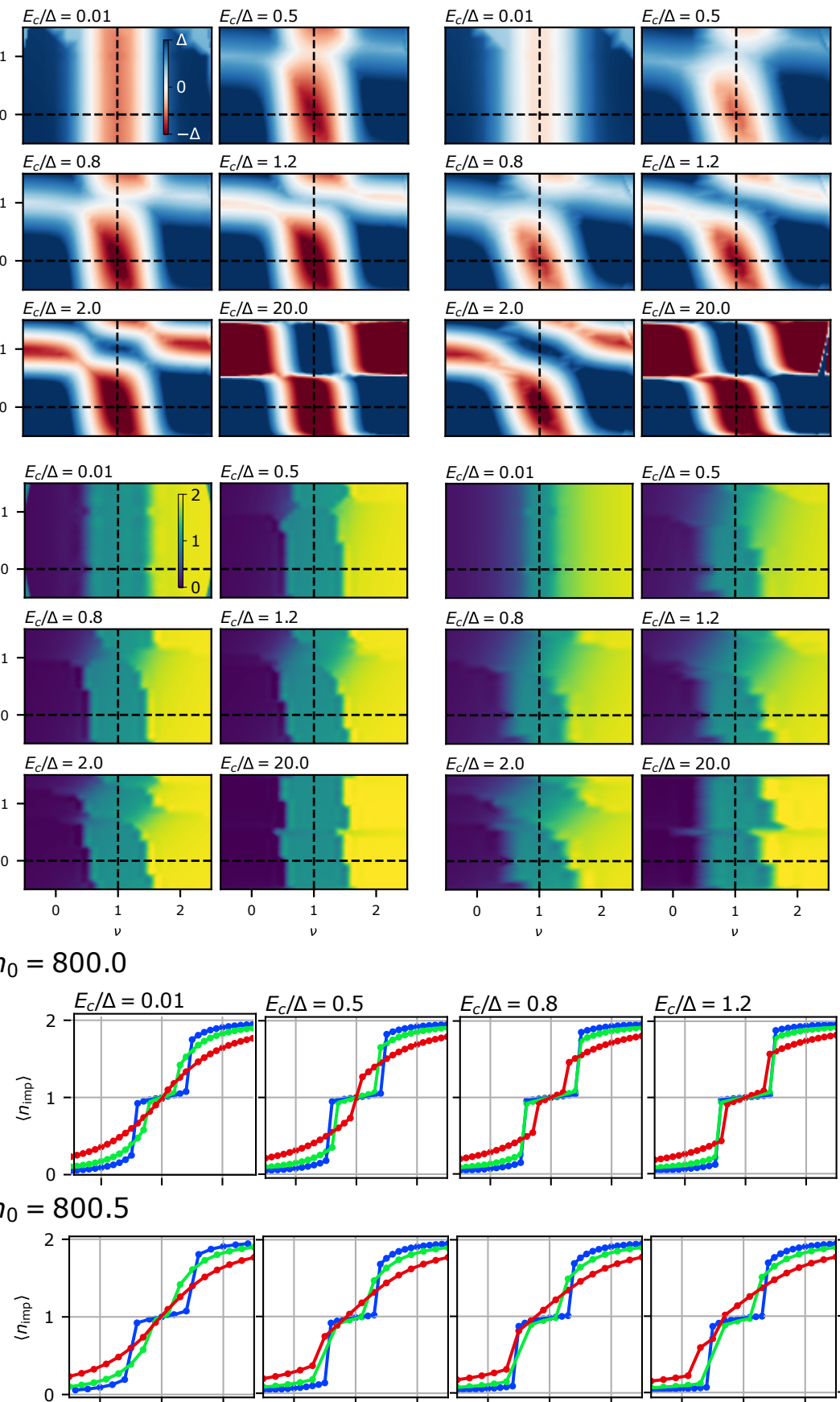

$E_{c} / \Delta=1.2$
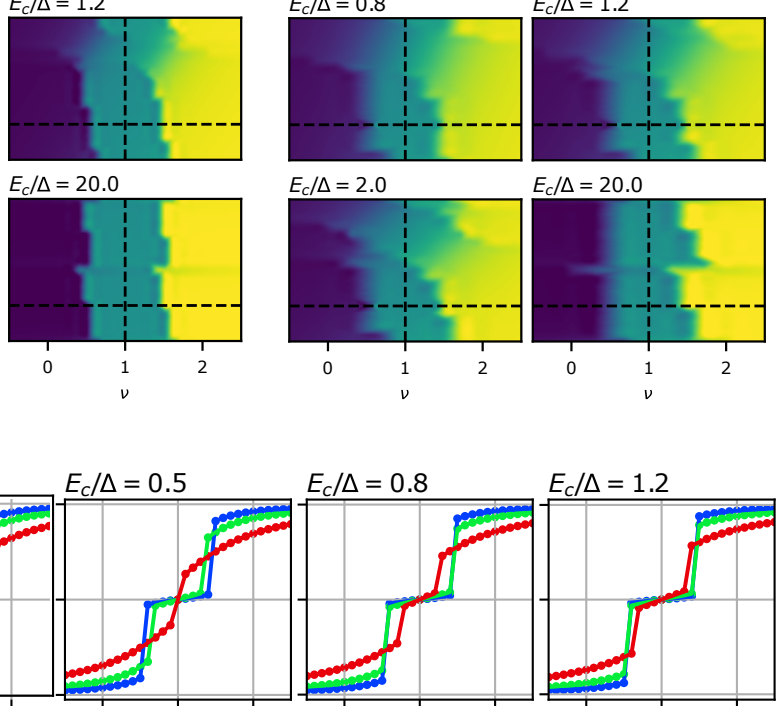

$\Gamma / U=0.5$
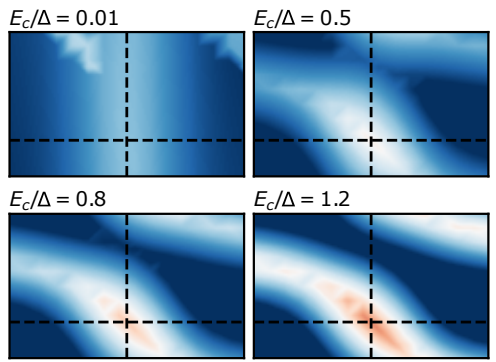

$E_{c} / \Delta=1.2$
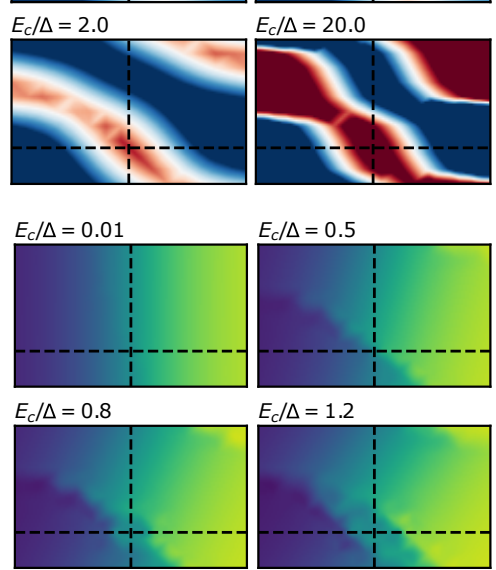

$E_{c} / \Delta=1.2$
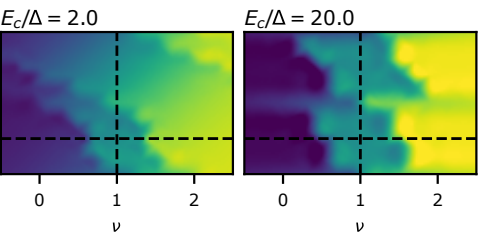

$n_{0}=801.0$
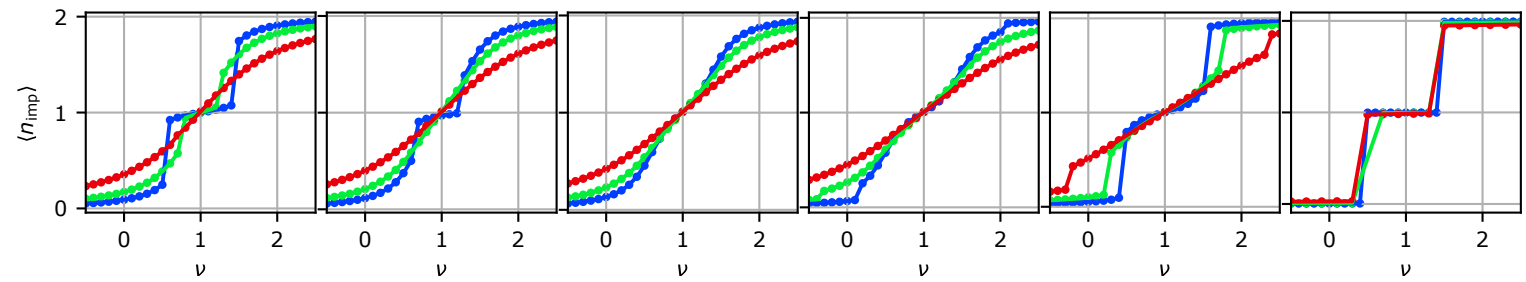

Figure S4. a) Charging diagrams for a range of $\Gamma / U . \Gamma / U=0.1$ is the case shown in Fig. 2 of the main text. b) Corresponding diagrams of the impurity occupancy, and c) cross-cuts at constant $n_{0}=800,800.5$, and 801 . 


\section{Spectral functions}

The subgap (discrete) part of the spectral function is easily computed from the wavefunctions of $(0),(+1)$ and (-1) states as

$$
\begin{aligned}
A_{\sigma}(\omega) & =\left|\left\langle\psi_{\sigma^{\prime}}^{(+1)}\left|d_{\sigma}^{\dagger}\right| \psi_{\sigma_{0}}^{(0)}\right\rangle\right|^{2} \delta\left[\omega-\left(E^{(+1)}-E^{(0)}\right)\right] \\
& +\left|\left\langle\psi_{\sigma^{\prime \prime}}^{(-1)}\left|d_{\sigma}\right| \psi_{\sigma_{0}}^{(0)}\right\rangle\right|^{2} \delta\left[\omega+\left(E^{(-1)}-E^{(0)}\right] .\right.
\end{aligned}
$$

Here $\sigma^{\prime}=\sigma_{0}+\sigma$ and $\sigma^{\prime \prime}=\sigma_{0}-\sigma$, where $\sigma_{0}$ is the $S_{z}$ component of total spin of the ground state (0), while $\sigma^{\prime}$ and $\sigma^{\prime \prime}$ are those of the excitations $(+1)$ and (-1), respectively.

In Fig. S5 we supplement the spectra shown in Fig. 3 of the main text with the additional results that reveal the nature of the relevant ground and excited states, and show the evolution with increasing $E_{c}$ leading to the $E_{c}>\Delta$ regime (Fig. 4 of the main text). Specifically, in Fig. S5 we compare the $\nu$ dependence of the subgap state energies, spectral weights, and the expectation values of occupancy, local moment, and spin-spin correlation for the range of $E_{c}$ discussed in the main text.

The left-most panels show the reference results for $E_{c}=$ 0, i.e. the conventional YSR regime. The model parameters used here correspond to the situation where close to half filling ( $\nu=1$, particle-hole symmetric point) the ground state is an (unscreened) doublet, while sufficiently away from half filling it is a singlet. At $\nu \sim 1$, the doublet GS with $\left\langle n_{\mathrm{imp}}\right\rangle \approx 1$ is characterized by a nearly saturated local moment $\left\langle\mathbf{S}_{\mathrm{imp}}^{2}\right\rangle \rightarrow 3 / 4$ that is almost decoupled from the band electrons, $\sum_{i}\left\langle\mathbf{S}_{\mathrm{imp}} \cdot \mathbf{S}_{i}\right\rangle \approx 0$. Sufficiently away from $\nu=1$, the singlet GS has level filling closer to either zero or full (double) occupancy and correspondingly diminished local moment $\left\langle\mathbf{S}_{\text {imp }}^{2}\right\rangle \lesssim 0.15$. The excited states in the central $\nu \sim 1$ region have level filling with a strong dispersion, and there is strong antiferromagnetic alignment of the local moment with the electrons in the band: this is a manifestation of the bonding of the Bogoliubov quasiparticle that generates these subgap YSR states. We note some small differences in the results for $(+1)$ and (-1) excited states (in particular the red and blue symbols do not overlap completely): this is a consequence of the finite size (finite $N$ ) of the system, as discussed earlier. For $E_{c}=0$ the results do not depend on $n_{0}$. The results are therefore (anti)symmetric with respect to $\nu=1$ for both even and odd $n_{0}$.

For finite but small $E_{c}=0.2 \Delta$, the main qualitative difference compared to $E_{c}=0$ is the observably different behavior of the (+1) and (-1) excitations, far exceeding the finite- $N$ effects we noted for the case of $E_{c}=0$. Furthermore, we observe a lack of (anti)symmetry with respect to $\nu=1$ for odd $n_{0}=801$. The nature of the states remains, however, the same as for $E_{c}=0$.

The regime of $E_{c} / \Delta=0.8$ and $E_{c} / \Delta=1.2$, where the electron-electron repulsion terms on the superconductor, $E_{c}$, and on the quantum dot, $U$, are comparable in magnitude (specifically $E_{c} /(U / 2) \approx 0.4,0.6$ ), is controlled by the competition between the QD and SC filling. In the following we analyse this regime in more details, separately for even and odd $n_{0}$.

For even $n_{0}$, close to the $\mathrm{p}$-h symmetric point $(\nu \approx 1)$, the ground state $(0)$ is a doublet with the impurity local moment almost decoupled from the SC. The excited states $( \pm 1)$ are similar to conventional YSR singlets, but with $n_{\text {imp }}$ considerably different from 1 due to charging terms.

Away from half filling, for large values of $\nu(\nu \gtrsim 1.5)$, in the state (0) there are almost 2 electrons on the impurity and $n_{\mathrm{sc}}$ is even. In (+1), the additional electron enters an empty SC level, which costs $E_{c}+\Delta$, thus $E^{+}$lies at the bottom of the continuum. To obtain (-1), the electron removed from the GS does not originate entirely from the SC but also partly from the impurity $\left(\left\langle n_{\mathrm{imp}}\right\rangle<2\right.$ in the hole-like excited state), recovering some of the local moment $\left\langle S_{\text {imp }}^{2}\right\rangle$ and shifting $E^{-}$inside the gap due to hybridisation.

For odd $n_{0}$, close to the p-h symmetric point the GS (0) and the excited states $( \pm 1)$ all have an occupancy close to 1 and a well developed local moment. The difference between these states consists in the fate of this moment: in the GS it forms a strong QD-SC singlet state with the lone Bogoliubov quasiparticle, while in $( \pm 1)$ states it is simply decoupled. The excitation energies are given by the sum of $-\Delta+E_{c}=0.2 \Delta$ and a contribution proportional to $J_{K}$, as discussed in the main text.

For large values of $\nu \sim 2$, the competition between $E_{c}$ and $U$ is very prominent and we need to distinguish the regions where the GS has 802 or 803 electrons; the transition between them occurs at a value of $\nu$ that strongly depends on $E_{c} / \Delta$. In the region with $n_{\mathrm{GS}}=802$, the state (0) is a singlet with high $n_{\text {imp. }}$. It has a large SC charging energy, while the impurity e-e repulsion energy $\frac{U}{2}\left(n_{\text {imp }}-\nu\right)^{2}$ is almost minimized. An additional electron predominantly enters the SC, hence the peak $E^{+}$has low spectral weight. The states (-1) and (0) are, however, quite similar, except for the additional electron at the impurity site in the state (0). The spectral weight of the $E^{-}$peak is thus large, on the order of 0.85 , and the corresponding excitation energy is large because the low-energy impurity level is emptied. In the region with $n_{\mathrm{GS}}=803$, the states $(0),(-1)$ and $(+1)$ all have close to maximal $n_{\text {imp }}$, and differ only in the electron close to the Fermi level in the SC, leading to extremely small spectral weights of the subgap peaks.

We note the existence of cases where the "subgap" state energy exceeds the $\Gamma=0$ gap edge, see Fig. S6 At $E_{c} / \Delta=0.2$ with odd $n_{0}$, the gap edge for $E_{c}<\Delta$ (thin dashed line) is given by $\Delta-E_{c}$ (see section below for derivation). In the bias voltage range indicated by the box in Fig. S6, the "subgap" state crosses this line with a finite weight of the spectral peak, in striking contrast to the usual situation where the YSR peaks transfer weight continuously as they approach the gap edge when the bound state merges with the continuum. This effect occurs away from $\nu=1$ for finite $E_{c}$ and odd $n_{0}$, in situations where 


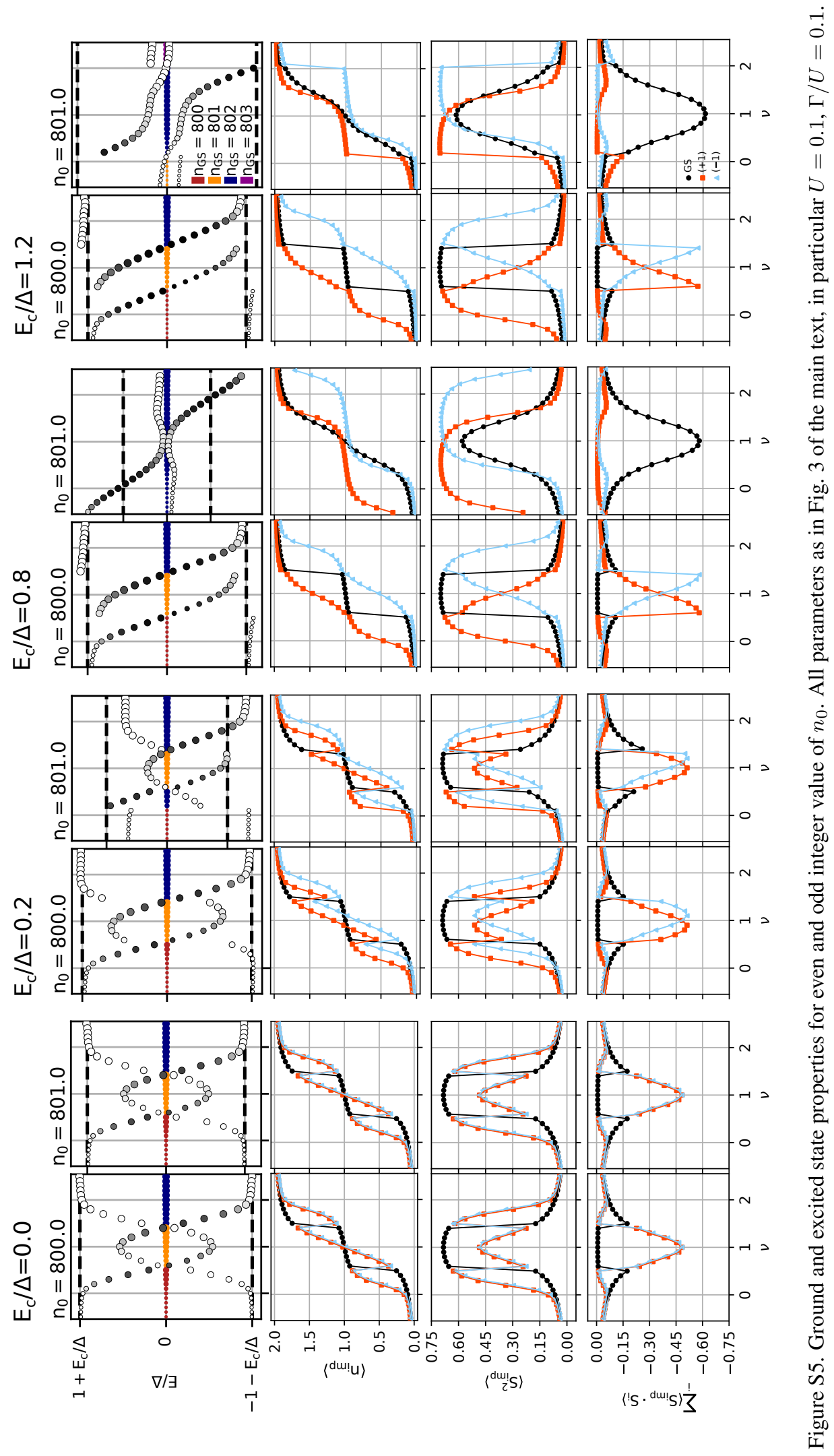




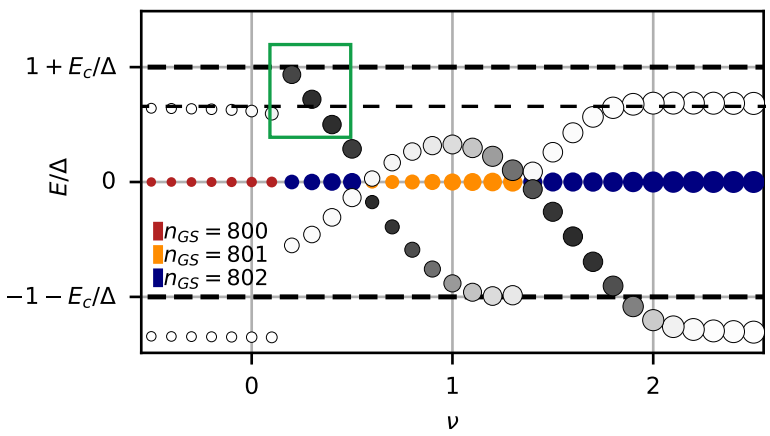

Figure S6. Spectral function for odd $n_{0}=801$ and $E_{c} / \Delta=0.2$, the case shown in Fig. 3f) in the main text. The thin dashed line at $\Delta-E_{c}$ shows the edge of the continuum for the isolated SC island $(\Gamma=0$ limit).

a)

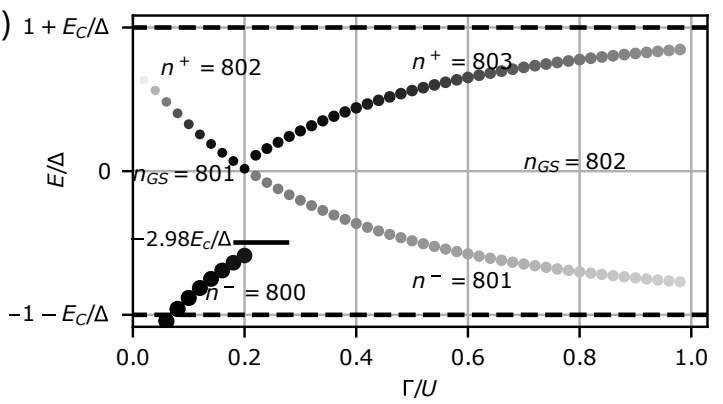

b)

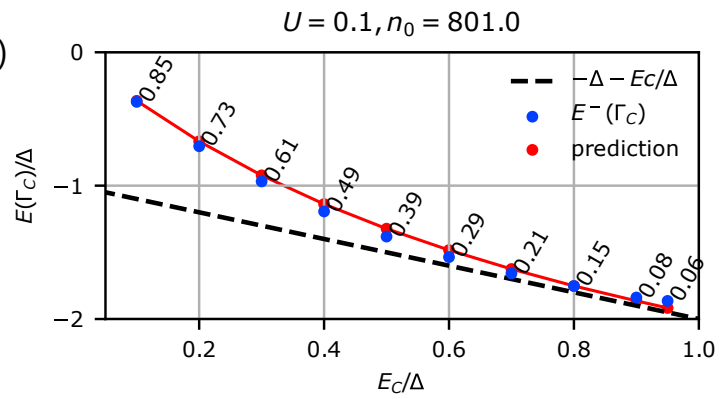

Figure S7. Quantifying $E_{c}$ from the discontinuities in spectral functions at odd SC filling, $n_{0}=801$. Top: spectral function as a function of hybridisation $\Gamma$, across the doublet-singlet transition. Here $U=0.1, E_{c} / \Delta=0.2, \nu=1$ (the case of Fig. 4 ). Bottom: discontinuity $E^{-}$as a function of $E_{c}$ (blue dots). Red line indicates an estimated based on electrostatic energy (see text) taking into account the impurity occupancy at the transition point (black labels). Dashed line indicates the gap edge. $U=0.1, \nu=1$.

it is advantageous for the tunneling electron to occupy the impurity orbital rather than enter a SC level.

\section{Extraction of $E_{c}$ from discontinuities}

The $\mathrm{p}$-h asymmetry and discontinuities provide a means to determine the charging energy $E_{c}$ from experimental spectra. This is best done for the system tuned to odd $n_{0}$ where the asymmetries are maximal. In Fig. S7(a) we plot the $\Gamma$-dependence of the peak positions for an impurity tuned to $\nu=1$. For $E_{c}=0, E^{(0)}, E^{(+1)}$ and $E^{(-1)}$ would all be equal at the doublet-single transition point $\Gamma=\Gamma_{c}$. For $E_{c}>0$, this no longer holds for odd $n_{0}$, as $n_{\mathrm{sc}}$ of (-1) differs from $n_{0}$. The asymmetry $E^{+}-E^{-}$is proportional to $E_{c}$, with a prefactor that depends on the impurity occupancy and in general needs to be determined numerically, see Fig. S7, b). In the large- $U$ (Kondo) limit where $n_{\text {imp }} \approx 1$, the energy difference is simply $4 E_{c}$ and $E_{c}$ could be directly extracted from experimental measurements. For comparable values of $E_{c}$ and $U$, this is no longer the case and the competition between the QD and SC charging terms is observed. In this case, $E^{+}-E^{-}$can be approximated by the difference of the sums of the impurity and SC charging energies in each state: $E^{+}-E^{-}=\left(E_{\mathrm{imp}}^{(+1)}+E_{c}\left(n_{s c}^{(+1)}-n_{0}\right)^{2}\right)-$ $\left(E_{\mathrm{imp}}^{(-1)}+E_{c}\left(n_{s c}^{(-1)}-n_{0}\right)^{2}\right)$, where $E_{\mathrm{imp}}^{x}$ is the expectation value of $H_{\mathrm{imp}}$ in the sector indicated by the superscript label $x \in\{(+0),(-1),(+1)\}$. This estimate is plotted in Fig. $\mathbf{S 7}$ as the red line labelled "prediction". The good agreement with the exact results indicates that these effects are indeed controlled mainly by the charging terms, while the hybridisation energy is roughly the same in $(+1)$ and $(-1)$. In order to experimentally determine $E_{c}$ in this regime, it is necessary to have either accurate information about the impurity occupancy, or make use of numerical calculations to fit the experimental results and extract the model parameters.

\section{Model definition}

Here we provide some further details on the Hamiltonian used in the main text. We first introduce the notation for level and electron numbers. $N$ is the number of levels in the SC, $M=N+1$ is the total number of levels in the problem. We furthermore define the following occupancy operators:

$$
\hat{n}_{\mathrm{imp}, \sigma}=d_{\sigma}^{\dagger} d_{\sigma}, \quad \hat{n}_{\mathrm{imp}}=\hat{n}_{\mathrm{imp}, \uparrow}+\hat{n}_{\mathrm{imp}, \downarrow},
$$

for the impurity, and

$$
\hat{n}_{\mathrm{sc}}=\sum_{i \sigma} c_{i \sigma}^{\dagger} c_{i \sigma}
$$

for the SC, as well as $\hat{n}=\hat{n}_{\text {imp }}+\hat{n}_{\mathrm{sc}}$. We write $n_{\text {imp }}=$ $\left\langle\hat{n}_{\mathrm{imp}}\right\rangle, n_{\mathrm{sc}}=\left\langle\hat{n}_{\mathrm{sc}}\right\rangle$ and $n=\langle\hat{n}\rangle$. Evidently, $n_{\mathrm{imp}}+n_{\mathrm{sc}}=$ $n$. At half filling $n_{\mathrm{imp}}=1, n_{\mathrm{sc}}=N$, and $n=1+N=$ $M$.

The SC parts of the Hamiltonian are

$$
\begin{aligned}
& H_{\mathrm{sc}}^{\prime}=\sum_{i, \sigma} \epsilon_{i} c_{i \sigma}^{\dagger} c_{i \sigma}-\alpha d \sum_{i, j}^{N} c_{i \uparrow}^{\dagger} c_{i \downarrow}^{\dagger} c_{j \downarrow} c_{j \uparrow}, \\
& H_{\mathrm{sc}}^{\prime \prime}=E_{c}\left(\hat{n}_{\mathrm{sc}}-n_{0}\right)^{2} .
\end{aligned}
$$


The pairing terms only include the time-reversal conjugate states, i.e., the Hamiltonian takes the form of the reduced pairing model (see also Appendix C in Ref. 27). $\alpha$ is the (dimensionless) strength of the attractive electron-electron interaction. $E_{c}$ is the charging energy of the SC island, $E_{c}=e_{0}^{2} / 2 C$, where $C$ is the total capacitance of the island. The interlevel spacing is $d=2 D / N$, where $2 D$ is the bandwidth of the conduction band. More precisely, the energy levels are $\epsilon_{i}=-D+d / 2+(i-1) d+x$ for $i=1, \ldots, N$, so that $\epsilon_{1}=-D+d / 2+x$ and $\epsilon_{N}=+D-d / 2+x$. Here $x=\alpha d / 2$ is a small shift that restores the p-h symmetry of the finite-size problem, as discussed in a later subsection.

The impurity part of the Hamiltonian is

$$
H_{\mathrm{imp}}=\sum_{\sigma} \epsilon_{\mathrm{imp}} \hat{n}_{\mathrm{imp}, \sigma}+U \hat{n}_{\mathrm{imp}, \uparrow} \hat{n}_{\mathrm{imp}, \downarrow}
$$

We introduce $\delta=\epsilon_{\text {imp }}+U / 2$, as well as $\nu=1 / 2-$ $\epsilon / U=1-\delta / U$, as two further ways to express the value of $\epsilon_{\mathrm{imp}}$ for a given fixed value of $U$. Both measure the departure from the p-h symmetric point at $\delta=0$ and $\nu=$ $1, \delta$ in energy units, $\nu$ in units of electron number. Thus, alternatively,

$$
\begin{aligned}
H_{\mathrm{imp}} & =\frac{U}{2}\left(\hat{n}_{\mathrm{imp}}-1\right)^{2}+\delta\left(\hat{n}_{\mathrm{imp}}-1\right)+\mathrm{const} \\
& =\frac{U}{2}\left(\hat{n}_{\mathrm{imp}}-\nu\right)^{2}+\mathrm{const} .
\end{aligned}
$$

Finally, the hybridisation part is

$$
H_{\text {hyb }}=\frac{v}{\sqrt{N}} \sum_{i, \sigma}^{N}\left(c_{i \sigma}^{\dagger} d_{\sigma}+\text { h.c. }\right) \text {. }
$$

We define $\Gamma=\pi \rho v^{2}$ where $\rho=1 / 2 D$ is the density of states in the conduction band. Conversely, $v=\sqrt{\Gamma / \pi \rho}=$ $\sqrt{2 \Gamma / \pi}$.

In a strictly electrically isolated QD-SC system the number of electrons would be fixed. The presence of weakly coupled tunneling probes permits the transfer of charge to and from the QD-SC system. The total occupancy changes so as to reach the state of minimal energy. (Strictly speaking the thermodynamic variable that is minimized is $H-\mu n$, but $\mu$ may be thought to be absorbed in the parameters $n_{0}$ and $\nu$ ). Nevertheless, for weak tunneling probe coupling the charge fluctuations may be neglected and the total QD-SC system charge does not change with time beyond the tunneling events when the system is probed. For this reason, one may take $\langle\hat{n}\rangle \equiv n$ to be an integer constant. Of particular interest is the parity of $n$ in the ground state. In the usual discussions of the YSR physics, where $E_{c}=0$ and $n_{\mathrm{imp}} \approx 1$, the odd-parity state (doublet state) corresponds to an unscreened impurity, the evenparity state (singlet state) to a "YSR screened" impurity. At finite $E_{c}$ this picture is modified by the additional energy shift of $E_{c}$ for states with SC occupancy differing by one electron.
We denote the lowest-energy eigenstate in each charge sector as $\psi^{n}$ and its energy as $E^{n}$. The ground state is thus $\psi^{n_{\mathrm{gs}}}$ and the lowest excited states $\psi^{n_{\mathrm{gs}}+1}$ and $\psi^{n_{\mathrm{gs}}-1}$. The excitation energies of the spectroscopically visible subgap states are defined as $E^{+}=E^{(+1)}-E^{(0)}$ for particle addition and $E^{-}=E^{(-1)}-E^{(0)}$ for particle removal. The corresponding spectral weights are $w^{+}=\left|\left\langle\psi^{(+1)}\left|d_{\sigma}^{\dagger}\right| \psi^{(0)}\right\rangle\right|^{2}$ and $w^{-}=\left|\left\langle\psi^{(-1)}\left|d_{\sigma}\right| \psi^{(0)}\right\rangle\right|^{2}$.

It is perhaps worthwhile to point out that since our model is based on a "microscopic" description of the pairing interaction and since the Hamiltonian is solved essentially exactly within the DMRG, the "gap renormalization effects" (the effect of the impurity back on the superconductor) is fully taken into account, thus no self-consistent correction of the pairing function is necessary as in mean-field approaches. This $1 / N$ renormalization effect is, however, small even for the $N=800 \mathrm{SC}$ levels used in the calculations in this work.

\section{Continuum edges}

For easy reference, let us consider the lowest particleaddition and particle-removal excitation energies for a pure $\mathrm{SC}$ island in the absence of the QD, i.e., the edges of the quasi-continua of Bogoliubov quasiparticles for finite $E_{c}$.

From Eq. (4) (for $\Gamma \equiv 0$ ), we find that for a GS with an even integer occupancy of the superconductor $n_{\mathrm{sc}}$

$$
\begin{aligned}
& E^{+}=\Delta+E_{c}+2 E_{c}\left(n_{\mathrm{sc}}-n_{0}\right), \\
& E^{-}=\Delta+E_{c}-2 E_{c}\left(n_{\mathrm{sc}}-n_{0}\right) .
\end{aligned}
$$

Here $n_{0}$ is the continuously tunable experimental parameter proportional to gate voltage, while $n_{\mathrm{sc}}=\left\langle\hat{n}_{\mathrm{sc}}\right\rangle$ is an integer (except at the charge degeneracy points). This reduces to $E^{+}=E^{-}=\Delta+E_{c}$ for even integer $n_{0}=n_{\mathrm{sc}}$, but one should note that $E^{+}$and $E^{-}$are shifted asymmetrically for any value of $n_{0}$ that is not an even integer. The total single-particle gap in the spectral function thus remains constant, $E^{+}+E^{-}=2 \Delta+2 E_{c}$. The largest asymmetry occurs for values close to odd $n_{0}$. Exactly at odd-integer $n_{0}, n_{\text {sc }}$ changes discontinuously by 2 for $E_{c}<\Delta$. On one side of this discontinuity one finds

$$
\begin{aligned}
& E^{+}=\Delta-E_{c}, \\
& E^{-}=\Delta+3 E_{c},
\end{aligned}
$$

and on the other

$$
\begin{aligned}
& E^{+}=\Delta+3 E_{c}, \\
& E^{-}=\Delta-E_{c} .
\end{aligned}
$$

For $E_{c}=0$ we recover the standard BCS result with the $\mathrm{SC}$ gap edges at $\omega=E^{+}=\Delta$ and $\omega=-E^{-}=-\Delta$ for all values of $n_{0}$. 
For large $E_{c}>\Delta$, the excitation gaps of the even $n_{\mathrm{sc}}$ states close at

$$
\begin{aligned}
& n_{0}=n_{\mathrm{sc}}+\frac{1}{2}\left(1+\Delta / E_{c}\right), \\
& n_{0}=n_{\mathrm{sc}}-\frac{1}{2}\left(1+\Delta / E_{c}\right),
\end{aligned}
$$

for particle-addition and particle-removal gap, respectively. In the range of $n_{0}$ where the GS has an odd number of electrons in the SC the following expressions hold:

$$
\begin{aligned}
& E^{+}=-\Delta+E_{c}+2 E_{c}\left(n_{\mathrm{sc}}-n_{0}\right), \\
& E^{-}=-\Delta+E_{c}-2 E_{c}\left(n_{\mathrm{sc}}-n_{0}\right) .
\end{aligned}
$$

For odd $n_{\mathrm{sc}}=n_{0}$ we thus find $E^{+}=E^{-}=-\Delta+E_{c}$.

In Fig. S8 we show a graphical overview of these results. For $E_{c} / \Delta=0,0.2,0.8,1.2$ (the values used in Fig. 3 of the main text) we plot in separate rows i) the energies of the SC states, ii) these same energies referred to the lowest (GS) energy, iii) the excitation energies $E^{+}$and $E^{-}$, iv) the edges of the continuum of single-particle (Bogoliubov) excitations at $\omega=+E^{+}$and $\omega=-E^{-}$.

\section{Particle-hole symmetry}

The particle-hole (p-h) transformation is defined as

$$
\begin{aligned}
& d^{\dagger} \rightarrow d, \\
& c_{i}^{\dagger} \rightarrow-c_{N+1-i} .
\end{aligned}
$$

The Hubbard and hopping terms remain invariant. The charge terms transform as $\hat{n}_{\mathrm{imp}} \rightarrow 1-\hat{n}_{\mathrm{imp}}, c_{i, \sigma}^{\dagger} c_{i, \sigma} \rightarrow$ $1-c_{N+1-i, \sigma}^{\dagger} c_{N+1-i, \sigma}$, so that $n_{\mathrm{sc}} \rightarrow N-n_{\mathrm{sc}}$. Finally, the pairing terms transform as $\sum_{i, j} c_{i \uparrow}^{\dagger} c_{i \downarrow}^{\dagger} c_{j \downarrow} c_{j \uparrow} \rightarrow$ $N-\sum_{i} \sum_{\sigma} c_{i \sigma}^{\dagger} c_{i \sigma}+\sum_{i, j} c_{i \uparrow}^{\dagger} c_{i \downarrow}^{\dagger} c_{j \downarrow} c_{j \uparrow}$. The Hamiltonian thus remains invariant if

$$
\begin{aligned}
\epsilon_{\mathrm{imp}} & =-U / 2, \\
\epsilon_{i} & =-\epsilon_{N+1-i}+g .
\end{aligned}
$$

The solution to the second equation for equidistant levels with spacing $d$ is

$$
\begin{aligned}
\epsilon_{i} & =-D+\frac{d}{2}+(i-1) d+\frac{\alpha d}{2} \\
& =-D+\left(i-\frac{1-\alpha}{2}\right) d .
\end{aligned}
$$

In the $N \rightarrow \infty$ limit, this converges to a flat band with the density of states $\rho=1 / 2 D$ on the interval $[-D: D]$.

\section{Truncation of the SC levels}

In Hamiltonian $H_{\mathrm{sc}}^{\prime}$, we truncate the spectrum of the $\mathrm{SC}$ levels at the Debye frequency $\omega_{D}$. For $\Gamma=0$ this is no approximation, because the levels outside the range $\left[-\omega_{D}: \omega_{D}\right]$ play no role since they are fully decoupled from the levels participating in pairing [36-40]. For $\Gamma \neq 0$, equating $D=\omega_{D}$ is an approximation, since the SC levels in ranges $\left[-D:-\omega_{D}\right]$ and $\left[\omega_{D}: D\right]$ are omitted. If required, one could explicitly take into account these noninteracting levels through the renormalization of model parameters [69] using, for example, the numerical renormalization group (NRG) method [10, 70]. An alternative correction scheme is to consider the cut-off $\omega_{D}$ to be increased to $D$, while the coupling constant $\alpha$ is decreased accordingly so that the gap $\Delta$, estimated through the BCS relation $\Delta=\omega_{D} \exp (-1 / \alpha d)$ remains constant. In any case, the approximation $\omega_{D}=D$ has no qualitative effect on the results.

Another observation is that typically the bath has a very large number of levels, while only a tiny subset of those is actually hybridized with the impurity. The effective quantum impurity problems with noninteracting baths fully disregard all levels which are decoupled from the impurity, because those live in a separate Hilbert space and are irrelevant for the solution of the impurity problem. In our work we also retain only the levels that hybridise with the impurity and neglect all others, but we need to keep in mind that in reality the Coulomb interaction connects the two subsystems which therefore do not fully decouple. We do not discuss effects resulting from such coupling in this work, but merely note that they are expected to be important for the transport properties.

\section{Implementation of the method}

We first provide the matrix-product-operator (MPO) representation of the Hamiltonian studied in this work. Left-most site (impurity-site):

$$
W_{0}=\left(\begin{array}{lll}
I & \epsilon_{\mathrm{imp}} \hat{n}_{\mathrm{imp}}+U \hat{n}_{\mathrm{imp}, \uparrow} \hat{n}_{\mathrm{imp}, \downarrow}-d_{\uparrow} F-d_{\downarrow} F+d_{\uparrow}^{\dagger} F+d_{\downarrow}^{\dagger} F \quad 0 \quad 00 & 0
\end{array}\right) .
$$

Here $F=(-1)^{n}$ is the local fermionic-parity operator, which gives phase of -1 if there is an odd number of electrons on the site. 

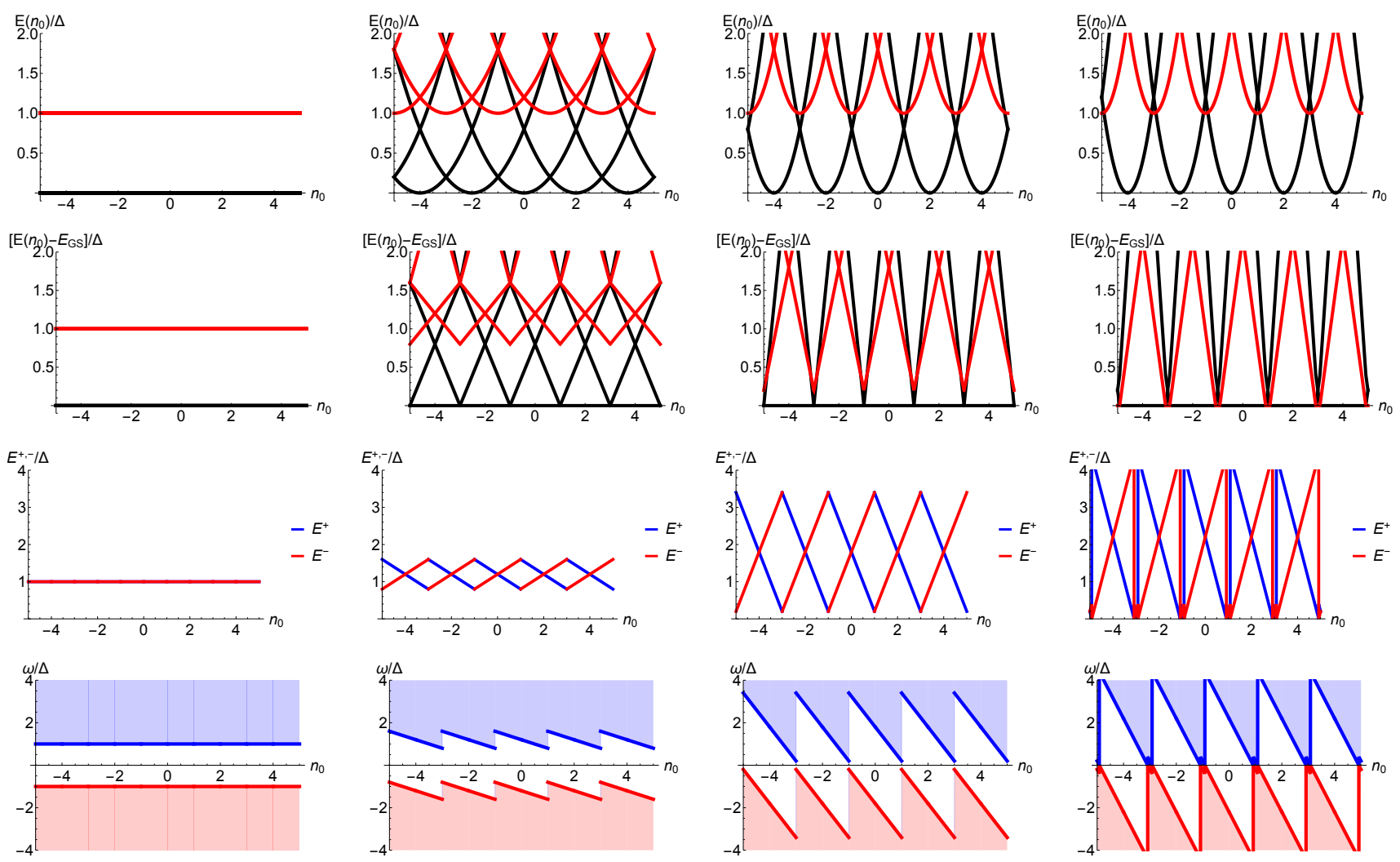

Figure S8. Single-particle excitation properties of the SC island in the absence of the QD, for (left to right) $E_{c} / \Delta=0,0.2,0.8,1.2$. In top rows, black stands for even $n_{\mathrm{sc}}$, red for odd $n_{\mathrm{sc}}$. For non-zero $E_{c}$, the charging has period $2 e$. For $E_{c} / \Delta<1$, the occupancy changes in steps of 2. For $E_{c} / \Delta>1$, the occupancy changes in steps of 1, yet the regions of even and odd occupancy have different widths and the $2 e$ period is maintained. With increasing $E_{c} / \Delta$, the even-odd effects become less pronounced; in the large $E_{c} / \Delta$ limit a 1e-periodic pattern typical of Coulomb blockade is recovered. At the same time, with increasing $E_{c} / \Delta$ the nature of the gap is changing from the superconducting gap into a Coulomb gap.

Generic site (with $g=\alpha d$ ):

$$
W_{i}=\left(\begin{array}{ccccccccc}
1 & {\left[\epsilon_{i}+E_{c}\left(1-2 n_{0}\right)\right] \hat{n}_{i}+\left(g+2 E_{c}\right) \hat{n}_{i \uparrow} \hat{n}_{i \downarrow}} & 0 & 0 & 0 & 0 & g c_{i \downarrow} c_{i \uparrow} & g c_{i \uparrow}^{\dagger} c_{i \downarrow}^{\dagger} & 2 E_{c} \hat{n}_{i} \\
0 & I & 0 & 0 & 0 & 0 & 0 & 0 & 0 \\
0 & v c_{i \uparrow}^{\dagger} & F_{i} & 0 & 0 & 0 & 0 & 0 & 0 \\
0 & v c_{i \downarrow}^{\dagger} & 0 & F_{i} & 0 & 0 & 0 & 0 & 0 \\
0 & v c_{i \uparrow} & 0 & 0 & F_{i} & 0 & 0 & 0 & 0 \\
0 & v c_{i \downarrow} & 0 & 0 & 0 & F_{i} & 0 & 0 & 0 \\
0 & c_{i \uparrow}^{\dagger} c_{i \downarrow}^{\dagger} & 0 & 0 & 0 & 0 & I & 0 & 0 \\
0 & c_{i \downarrow} c_{i \uparrow} & 0 & 0 & 0 & 0 & 0 & I & 0 \\
0 & \hat{n}_{i} & 0 & 0 & 0 & 0 & 0 & 0 & I
\end{array}\right),
$$

with

$$
\hat{n}_{i \sigma}=c_{i \sigma}^{\dagger} c_{i \sigma}, \quad \hat{n}_{i}=\sum_{\sigma} \hat{n}_{i \sigma}
$$

and $F_{i}$ is again a local parity operator. 
Right-most site:

$$
W_{N}=\left(\begin{array}{c}
{\left[\epsilon_{N}+E_{c}\left(1-2 n_{0}\right)\right] \hat{n}_{N}+\left(g+2 E_{c}\right) \hat{n}_{N \uparrow} \hat{n}_{N \downarrow}} \\
I \\
v c_{N \uparrow}^{\dagger} \\
v c_{N \downarrow}^{\dagger} \\
v c_{N \uparrow} \\
v c_{N \downarrow} \\
c_{N \uparrow}^{\dagger} c_{N \downarrow}^{\dagger} \\
c_{N \downarrow} c_{N \uparrow} \\
\hat{n}_{N}
\end{array}\right) .
$$

An alternative representation is possible where the impurity is located in the center of the 1D chain (corresponding to the Fermi level of the superconducting island) rather than attached to the end of the chain. We find fully equivalent results with both approaches, with rather similar bond dimensions (which are maximal in the vicinity of the Fermi level), similar to what has been observed in solving impurity models in the star geometry using the DMRG method [71].

The DMRG calculations have been performed using the ITensor library. The initial state is the Fermi sea with all lowlying levels of the SC occupied by electrons, and an additional electron on the impurity site. A low truncation criterion (sum of discarded Schmidt values) $\epsilon=10^{-12}$ and bond dimensions up to 5000 are required in order to reach convergence for $N=800$.

The symmetries exploited in the DMRG calculations were the charge conservation $U(1)$ and the spin conservation $U(1)$. The calculations were performed for $S_{z}=0$ in even-occupancy sectors and for $S_{z}= \pm 1 / 2$ in odd-occupancy sectors.

We note that our method is very different from that in Ref. 25] which is a momentum-space DMRG in the space of electron pairs ("particle-hole" method), and hence inapplicable to our Hamiltonian that explicitly breaks electron pairs through exchange processes.

\section{Simplification to $8 \times 8$-dimensional MPOs}

We now consider the parts of the Hamiltonian which control the occupancy, specifically:

$$
H^{\prime}=U \hat{n}_{\mathrm{imp}, \uparrow} \hat{n}_{\mathrm{imp}, \downarrow}+\epsilon \hat{n}_{\mathrm{imp}}+E_{c}\left(\hat{n}_{\mathrm{sc}}-n_{0}\right)^{2} .
$$

We note that

$$
\begin{aligned}
\left(\hat{n}_{\mathrm{sc}}-n_{0}\right)^{2} & =\left[\left(\hat{n}-\hat{n}_{\mathrm{imp}}\right)-n_{0}\right]^{2} \\
& =\left[\left(\hat{n}-n_{0}\right)-\hat{n}_{\mathrm{imp}}\right]^{2} \\
& =\left(\hat{n}-n_{0}\right)^{2}-2\left(\hat{n}-n_{0}\right) \hat{n}_{\mathrm{imp}}+\hat{n}_{\mathrm{imp}}^{2}
\end{aligned}
$$

and

$$
\begin{aligned}
\hat{n}_{\mathrm{imp}}^{2} & =\left(\hat{n}_{\mathrm{imp}, \uparrow}+\hat{n}_{\mathrm{imp}, \downarrow}\right)^{2} \\
& =\hat{n}_{\mathrm{imp}, \uparrow}^{2}+\hat{n}_{\mathrm{imp}, \downarrow}^{2}+2 \hat{n}_{\mathrm{imp}, \uparrow} \hat{n}_{\mathrm{imp}, \downarrow} \\
& =\hat{n}_{\mathrm{imp}, \uparrow}+\hat{n}_{\mathrm{imp}, \downarrow}+2 \hat{n}_{\mathrm{imp}, \uparrow} \hat{n}_{\mathrm{imp}, \downarrow} \\
& =\hat{n}_{\mathrm{imp}}+2 \hat{n}_{\mathrm{imp}, \uparrow} \hat{n}_{\mathrm{imp}, \downarrow},
\end{aligned}
$$

so that

$\left(n_{\mathrm{sc}}-n_{0}\right)^{2}=\left(\hat{n}-n_{0}\right)^{2}+\left[1-2\left(\hat{n}-n_{0}\right)\right] \hat{n}_{\mathrm{imp}}+2 \hat{n}_{\mathrm{imp}, \uparrow} \hat{n}_{\mathrm{imp}, \downarrow} \cdot$

Thus

$$
\begin{aligned}
H^{\prime} & =\left(U+2 E_{c}\right) \hat{n}_{\mathrm{imp}, \uparrow} \hat{n}_{\mathrm{imp}, \downarrow} \\
& +\left[\epsilon-2 E_{c}\left(\hat{n}-n_{0}\right)+E_{c}\right] \hat{n}_{\mathrm{imp}} \\
& +E_{c}\left(\hat{n}-n_{0}\right)^{2} .
\end{aligned}
$$

In the canonical ensemble we may replace $\hat{n}$ by $n$ in each charge sector. Thus the effective $U$ increases by $2 E_{c}$, the level is shifted by $-2 E_{c}\left(n-n_{0}\right)+E_{c}$, i.e. $\delta=\epsilon+U / 2$ is shifted by $-2 E_{c}\left(n-n_{0}\right)+2 E_{c}=-2 E_{c}\left[n-\left(n_{0}+\right.\right.$ $1)$ ], and the energy shift term becomes a constant, $E_{c}(n-$ $\left.n_{0}\right)^{2}$. We may thus eliminate the quadratic charge terms in the SC, while the impurity terms are renormalized. This is convenient for implementation and permits the reduction of the MPO representation to $8 \times 8$ matrices, however this form is less physically transparent.

\section{Benchmark calculations}

We verified the implementation at $\Gamma=0, E_{c}>0$ against the exact solution for the model without the impurity [38], finding full agreement within numerical roundoff errors for energies.

We verified the implementation at $\Gamma>0, E_{c}=0$ by comparing the results of numerical renormalization group (NRG) calculations for mean-field BCS bath (parametrized by the BCS gap value $\Delta$ ) and the DMRG calculations for interacting bath (parametrized by the pairing coupling constant $\alpha$ ). The NRG calculations are performed in the thermodynamic limit but for a logarithmically discretized bath, while the DMRG calculations are performed for large but finite number of levels $N$. We remark that the NRG is not exact (due to truncation of states, which leads to unavoidable systematic errors in addition to those due to logarith- 
mic discretization), while the DMRG has no systematic errors. Typical NRG errors for quantities such as excitation energies are of the order of few percent [72, 73]. We find that after the $N \rightarrow \infty$ extrapolation of the DMRG results for the excitation energies of the subgap states, we recover the NRG results within the expected error margin of a few percent, see Fig. S9 To obtain a mapping between $\Delta$ and $\alpha$, we performed the NRG calculations for a range of $\Delta$ and selected the value where the agreement of the YSR excitation energies was optimal. Since the value of the coupling constant $\alpha$ used in this work, $\alpha=0.23$, lies at the boundary between weak and strong-coupling BCS regimes, this empirical approach is more reliable than various analytical estimates for $\Delta$ as a function of $\alpha$.

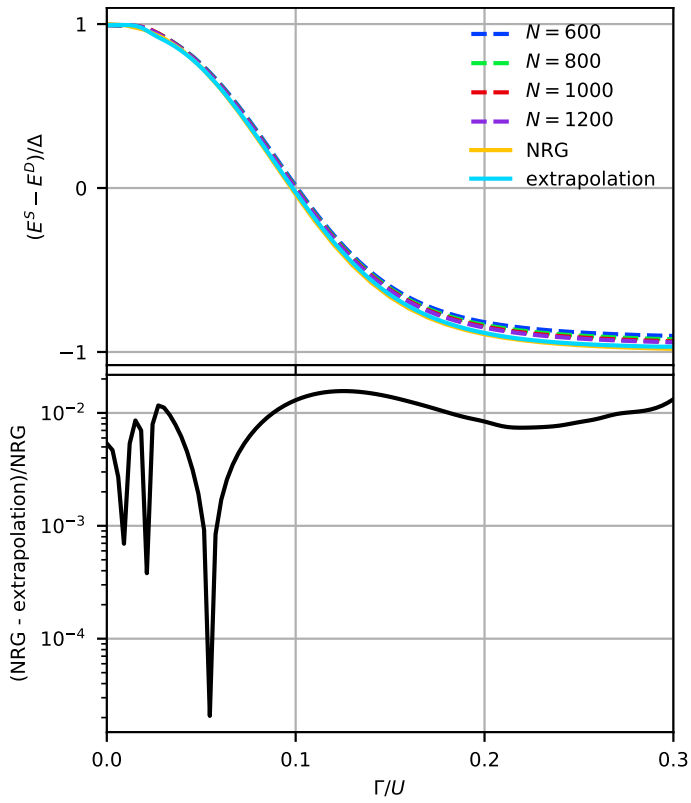

Figure S9. Benchmark calculations for $E_{c}=0$. (a) DMRG results for the YSR state energy $E_{\mathrm{YSR}}=E_{S}-E_{D}$ as a function of $\Gamma$ for a range of $N$ and the $N \rightarrow \infty$ extrapolation, compared against the results obtained using the numerical renormalization group (NRG) in the thermodynamic limit at the mean-field level. (b) Difference between the extrapolated DMRG results and the NRG results.

We furthermore tested the implementation with all terms of the Hamiltonian, including the charging terms with $E_{c} \neq 0$, against full diagonalisation on small clusters (up to $N=12$ ) using the Lanczos method, finding full agreement within the numerical roundoff errors. 